\title{
Symmetry breaking in Laughlin's state on a cylinder
}

\author{
S. Jansen ${ }^{1}$, E. H. Lieb ${ }^{2}$, R. Seiler ${ }^{1}$ \\ 1 Institut für Mathematik, Technische Universität Berlin, Str. des 17. Juni 136, 10623 Berlin, \\ Germany \\ 2 Department of Mathematics, Princeton University, P. O. Box 708, Princeton, NJ 08544, \\ USA
}

\begin{abstract}
We investigate Laughlin's fractional quantum Hall effect wave function on a cylinder. We show that it displays translational symmetry breaking in the axial direction for sufficiently thin cylinders. At filling factor $1 / p$, the period is $p$ times the period of the filled lowest Landau level. The proof uses a connection with one-dimensional polymer systems and discrete renewal equations.
\end{abstract}

\section{Contents}

1. Introduction .......................... 1

2. A solvable model . . . . . . . . . . . . . . . . . 6

3. Laughlin's cylinder function . . . . . . . . . . . . . 11

3.1 Basic properties . . . . . . . . . . . . . . . 12

3.2 Associated polymer system and renewal equation . . . . . . . . 14

3.3 Large $N$-asymptotics of normalization constants . . . . . . . 19

3.4 Thermodynamic limits of correlation functions and symmetries . 23

3.5 Symmetry breaking and clustering . . . . . . . . . . 27

\section{Introduction}

Our goal in this paper is to investigate the properties of a quantum mechanical wave function of very many variables known as the Laughlin function Lau2. It arises as an explanation of a curious and unexpected phenomenon known as the fractional quantum Hall effect (FQHE) [TSG].

Correspondence to: Sabine Jansen, E-mail: jansen@math.tu-berlin.de

(C) 2008 by the authors. This paper may be reproduced, in its entirety, for non-commercial purposes. 
It is not our intention to discuss the physics of FQHE but rather to discuss the mathematical problems that arise when the Laughlin function is appropriately modified for a two-dimensional cylindrical geometry [T. The interesting point here is that although the setup is seemingly translation invariant (in the direction of the cylinder axis) the resulting one-particle density $\rho$ is not invariant but has a non-trivial periodicity of length $p$ (in natural units), where $p$ is an integer related to the so-called "filling fraction". The main content of this paper is the rigorous verification of this periodicity when the cylinder radius is small compared to the natural length. The periodicity has a natural interpretation in the context of classical Coulomb systems and is manifest in the periodicity of a closely related "quantum polymer" JLS. The conjecture of periodicity for all radii remains open.

Let us just say a few words about the function and why it was proposed. Imagine a hollow circular cylinder in $\mathbb{R}^{3}$ of radius $R$ on whose surface $N$ electrons reside. Each electron has a coordinate $(x, y)$, with $x \in \mathbb{R}$ denoting the distance parallel to the cylinder axis and $y \in[0,2 \pi R)$ denoting the angular coordinate. We will often use $z=x+\mathrm{i} y$ to denote the coordinate instead of $(x, y)$. There is a magnetic field of magnitude $\boldsymbol{B}=\operatorname{curl} \boldsymbol{A}$ perpendicular to the surface. Since magnetic fields must be divergence free we can think of this situation as one in which there is a (unobserved) flux through the hollow cylinder that leaks out through the surface with a constant flux through the cylindrical surface on which the electrons reside (see also $[\mathrm{WH}$, Sect. III). If we pay attention only to the surface the physical situation is translation invariant but if we pay attention to the flux in the hollow core we can measure our $x$ coordinate by measuring the total flux in the circular cross section at $x$. The magnetic vector potential, $\boldsymbol{A}$, evaluated on the surface, is given by Stokes' theorem (assuming that $\boldsymbol{A}$ has no component in the axial direction) as $\boldsymbol{A}(x, y)=(0, B x+\phi /(2 \pi R))$, which is obviously periodic in the $y$ direction. Our vector potential $\boldsymbol{A}(z)$ is determined only up to a gauge parametrized by $\phi$.

With this choice the one particle Schrödinger operator is

$$
H=\frac{1}{2}(\boldsymbol{p}-\boldsymbol{A}(z))^{2}=\frac{1}{2}\left(\left(-i \partial_{x}\right)^{2}+\left(-i \partial_{y}-B x-\frac{\phi}{2 \pi R}\right)^{2}\right)
$$

In our units Planck's constant $\hbar$, the mass and the charge of the electron are 1. To simplify notation $B$ actually denotes the magnetic field divided by the speed of light, and in the following we will choose units in such a way that $B=1$. Our operator $H$ should be seen as the part of a three-dimensional Hamiltonian which acts on the axial and the angular parts of the wave function 1 The vector potential $\boldsymbol{A}$ is the restriction to the cylinder surface of some three-dimensional vector potential that gives rise to a magnetic field as sketched in $[\mathrm{WH}$, Fig. 3.

In the chosen units, the "magnetic length" $\ell=(\hbar / e B)^{1 / 2}$ equals 1 . An important role will be played by the dimensionless parameter

$$
\gamma=\ell / R=1 / R
$$

\footnotetext{
1 In cylindrical coordinates, with $r, \theta, x$ the radial, angular and axial coordinates, the part of the Laplacian that we are interested in is $r^{-2} \partial_{\theta}^{2}+\partial_{x}^{2}$. Fixing $r=R$ and choosing $y=R \theta$, this becomes $\partial_{y}^{2}+\partial_{x}^{2}$ : the differential symbol is $R$-independent.
} 
Here we come to the crucial point that the usual gauge invariance (meaning that we can replace $\boldsymbol{A}(z)$ by $\boldsymbol{A}(z)+\nabla \tilde{\phi}(z)$ and a wave function $\Psi(z)$ by $\Psi(z) \exp [\mathrm{i} \tilde{\phi}(z)])$ is not generally allowed. Gauge changes $\exp (\mathrm{i} \tilde{\phi}(z))$ have to respect the $2 \pi R$-periodicity in the $y$ direction. Consequently, the number $\phi$ in $H$ cannot be changed by a gauge transformation unless the change is a multiple of $2 \pi$.

This lack of complete gauge symmetry is intimately related to the breaking of translational symmetry. If we translate in the axial direction by $\delta$ then $x \rightarrow x+\delta$, the Hamiltonian (11) with this replacement is generally not gauge equivalent to the original one. That is, changing $\boldsymbol{A}$ by $\nabla \tilde{\phi}$ with $\tilde{\phi}=\delta y$ is not allowed - except for the special cases $\delta=n / R=n \gamma$ with $n \in \mathbb{Z}$. Hence the system possesses a discrete translational symmetry.

The discrete translational invariance and the effect of changing $\phi$ can be nicely read off the eigenfunctions of $H$. The eigenfunctions belonging to the lowest eigenvalue are all of the form

$$
\psi(z)=f(z) \exp \left[-\left(x+\frac{\phi \gamma}{2 \pi}\right)^{2} / 2\right]
$$

where $f$ is an entire function. To respect $2 \pi R$ periodicity, the eigenfunction must be a superposition of functions

$$
\exp (\gamma n z) \exp \left[-\left(x+\frac{\phi \gamma}{2 \pi}\right)^{2} / 2\right] \propto \exp (\mathrm{i} n \gamma y) \exp \left[-\left(x-\left(n-\frac{\phi}{2 \pi}\right) \gamma\right)^{2} / 2\right],
$$

with $n \in \mathbb{Z}$. These functions are essentially Gaussians of $x$; their centers form a lattice with spacing $\gamma$, and changing $\phi$ amounts to a uniform shift of the centers. From now on, we set the parameter $\phi$ to 0 .

If we now consider a finite cylinder of length $L$ then we impose the 'physical' boundary condition that we restrict functions to the interval $0 \leq x \leq L$ and consider only ground state eigenfunctions with centers inside that interval 2 Thus, for a finite cylinder, the dimension of this lowest energy space - known as the lowest Landau band - equals the "number of fluxes" $R L$, i.e., the total flux $2 \pi R L B$ divided by the flux quantum $2 \pi$ (remember $B=1$ ).

If there are some number $N$ of electrons that repel each other via a Coulomb interaction we have to construct a wave function of $N$ variables $z_{1}, z_{2}, \ldots, z_{N}$ that minimizes the total energy. The kinetic part is minimal if we choose sums of products of one-particle functions from the lowest Landau band. In other words our wave function must have the form

$$
P\left(\exp \left(\gamma z_{1}\right), \exp \left(\gamma z_{2}\right), \ldots, \exp \left(\gamma z_{N}\right)\right) \exp \left(-\sum_{j=1}^{N} x_{j}^{2} / 2\right)
$$

where $P$ is a polynomial in $N$ variables of degree at most $R L$ in each variable separately. Furthermore, the Pauli exclusion principle for fermions demands that this polynomial be antisymmetric, i.e., that it change sign if any two variables are interchanged. If the particles are bosons, instead, then $P$ must be symmetric.

2 These are the ground state eigenfunctions of the Hamiltonian (1) with chiral boundary conditions AANS. 
We are interested in the cases that $N=R L / p$ with $p=1,2,3, \ldots$, in which case we say that the lowest Landau band is filled to a fraction $1 / p$. The function

$$
\Psi_{N}\left(z_{1}, \ldots, z_{N}\right)=\kappa_{N} \prod_{1 \leq j<k \leq N}\left(\exp \left(\gamma z_{k}\right)-\exp \left(\gamma z_{j}\right)\right)^{p} \exp \left(-\sum_{j=1}^{N} x_{j}^{2} / 2\right)
$$

satisfies all the stated conditions for fermions (when $p$ is odd) and for bosons (when $p$ is even). Later, it will be useful to fix the multiplicative factor $\kappa_{N}$ as in Eq. (17) below. The factor $\kappa_{N}$ does not affect the one-particle density. Our choice of $\kappa_{N}$ makes $\Psi_{N}$ the power of a Slater determinant of functions that resemble the lowest Landau level basis functions (see Eq. (44)), and considerably simplifies subsequent statements on normalization constants.

This function was invented by Laughlin Lau2 for the disk geometry and later adapted to the cylinder geometry [T], and is considered to be a good approximation to the true ground state. Its distinctive feature is that it is small when two particles $z_{j}$ and $z_{k}$ are close together, thereby making the interaction energy small.

In this article, we analyze the periodicity in the axial direction of the state $\left|\Psi_{N}\right\rangle\left\langle\Psi_{N}\right|$ in the limit $N \rightarrow \infty$ for fixed radius $R$ and filling factor $1 / p$. Particular emphasis will be put on the one particle density

$$
\rho_{N}(z)=\frac{N}{C_{N}} \int_{\mathbb{R}^{N-1}} \int_{\left[0,2 \pi \gamma^{-1}\right]^{N-1}}\left|\Psi_{N}\left(z, z_{2}, . ., z_{N}\right)\right|^{2} \mathrm{~d} y_{2} . . \mathrm{d} y_{N} \mathrm{~d} x_{2} . . \mathrm{d} x_{N},
$$

where $C_{N}=\left\|\Psi_{N}\right\|^{2}$ is the $L^{2}$-norm squared with respect to integration over $(\mathbb{R} \times$ $\left.\left[0,2 \pi \gamma^{-1}\right]\right)^{N}$. Notice that we integrate over infinite cylinders but keep referring to $\Psi_{N}$ as a finite cylinder state. The reason is that the wave function vanishes exponentially fast outside the finite cylinder. For $p=1$, the wave function is a simple Slater determinant of basis functions (2), the density is a sum of equally weighted Gaussians exp $\left[-(x-n \gamma)^{2}\right]$ (see Eq. (23)), and changing the domain of integration to a finite cylinder does not affect the density in the middle of the cylinder.

The origin of the analysis of the density's periodicity is an observation for the filled lowest Landau level: for $p=1$, the density is periodic with minimal period $\gamma$. This contrasts with the constant density of the filled Landau level in the symmetric gauge and reflects the discrete translational symmetry of the Hamiltonian (1).

When $p>1$, Laughlin's state does not possess the full discrete symmetry of the Hamiltonian (11) any longer, hence the symmetry is broken. However, the state is still periodic, with minimal period $p \gamma$, i.e., $p$ times the period of the filled Landau level. This is rigorously shown here for cylinders with sufficiently small radius (large $\gamma$ ); numerical results [کSWK] for $p=2$ and $p=3$ suggest that the result actually holds for all values of the cylinder radius. Related, finite $N$ results have been obtained in $[\mathrm{RH}]$. Related considerations for Laughlin type functions on tori, introduced in $[\mathrm{HR}$, can be found in [SFL, BK]. In [SFL, the amplitude of oscillations of the density for torus Laughlin-type functions is investigated numerically, as a function of the cylinder radius.

It follows from our results that the density and hence, the amplitude of $3 \gamma$ oscillations, are analytic function of $\gamma$ when $\gamma$ is sufficiently large. This implies 
that the only possibility for the oscillation amplitude to vanish on some small $\gamma$ interval is that a phase transition occurs as $\gamma$ is decreased, see $\underline{K}$ for a similar argument. (With "phase transition" we mean that either there is no longer a well-defined unique thermodynamic limit or there is a unique thermodynamic limit but the density ceases to be analytic at some point.) [BK] argue that no such phase transition occurs, but a rigorous proof is still lacking.

Due to the picture mentioned before, translation along the cylinder axis can be reinterpreted as a change of flux $\phi$. One flux unit $(2 \pi)$ corresponds to a translation by one natural length unit $(\gamma)$. Hence the many electron system is invariant under a change of $p$ flux units. In the picture of Laughlin's original article on the integer Hall effect Lau1 this corresponds to a transport of one electric charge from one edge to the other of the cylinder for every adiabatic change by $p$ flux units. This is related to a heuristic argument given in TW], suggesting that the periodicity is needed in order to reconcile Laughlin's IQHE article with fractional quantum Hall conductances.

Another interpretation of the periodicity is that the $N$ particles in a cylinder of length $p N$ are not arranged randomly but, more like a one-dimensional crystal, they are separated by a distance of $p$ units. This interpretation is of interest from the point of view of Laughlin's plasma analogy [Lau2: $\left|\Psi_{N}\right|^{2}$ is proportional to the Boltzmann weight of a classical jellium system placed on a cylinder (see also [JLS, Sect. 3). The factor $\exp \left(-\sum_{j} x_{j}^{2} / 2\right)$ in the wave function arises from the potential created by a neutralizing background while the polynomial in $\exp \left(\gamma z_{j}\right)$ accounts for the repulsive interaction between charged particles. One-dimensional jellium systems always display periodicity, i.e., "Wigner crystallization", as was shown in $\underline{\mathrm{K}} \mathrm{BL}$. For jellium tubes, the symmetry breaking is well-known at coupling constant $\Gamma=2$, corresponding to the filled Landau level, see CFS JL. The periodicity has been conjectured to hold for other values of the coupling constant [AGL. Our results give a partial proof of this conjecture.

Our result on the periodicity comes in several parts: a preliminary observation (Lemma 2), holding for all values of $\gamma$, is that the one particle density can never be constant, and if it is periodic, the period must be a multiple of $\gamma$. Theorem 2 , valid for large $\gamma$, states that the Laughlin state has a unique thermodynamic limit as $N \rightarrow \infty$, and the limiting state has $p \gamma$ as one of its periods. Theorem 3 says that $p \gamma$ is actually the smallest period. We also prove, for large $\gamma$, that the state is mixing with respect to (magnetic) translations along the direction of the cylinder axis (Theorem 4).

The crucial idea for the proof of our results is the representation of Laughlin's wave function as a "quantum polymer" [JLS]. This means that $\Psi_{N}$ is written as a sum of functions, each associated with a partition of $\{0, . ., N-1\}$. One of these functions is the Tao-Thouless function [TT, which is the antisymmetrized tensor product of lowest Landau band functions centered at multiples of $p \gamma$. The Tao-Thouless function can be interpreted as a simple monomer, see Fig. 1 The polymer expansion is explained in Subsects. 3.1 and 3.2. The associated polymer system has a built-in form of translational invariance that is at the origin of the $p \gamma$-periodicity in the limit $N \rightarrow \infty$.

One consequence of the polymer representation is a recurrence relation for the normalization constant $C_{N}$, known as a (discrete) renewal equation. In Subsect. 3.3. we show that the associated renewal process has finite mean for suffi- 


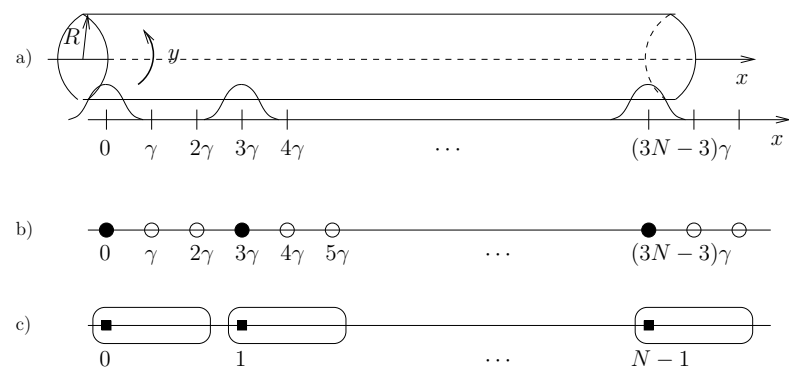

Fig. 1. a) The Tao-Thouless state for $p=3$ and $N$ particles is made up of lowest Landau band functions 2 with Gaussian centers $0,3 \gamma, \ldots,(3 N-3) \gamma$. b) This is visualized as a succession of $N$ times the sequence "filled circle - white circle - white circle". c) These $N$ blocks are associated with a monomer partition of $\{0, \ldots, N-1\}$.

ciently large $\gamma$. We use this important technical result in Subsects. 3.4 and 3.5 to prove our results on Laughlin's function.

At this point, let us mention that plasma analogy sheds some light not only on the density's periodicity, but also on the recurrence relation: indeed, in the context of jellium systems, a renewal equation already appeared in Len, while a renewal inequality showed up in LL.

In Sect. 2, we investigate a solvable model that arises as a simplification of Laughlin's function. In this model, only monomer-dimer systems occur. This allows us to introduce in a simpler setting the ideas described above.

\section{A solvable model}

As a preparation to the examination of Laughlin's wave function, we analyze a modified wave function. It is derived from Laughlin's wave function by replacing Gaussians with functions of compact support. If the support is small, the normalization constants and the one-particle density can be computed explicitly (Prop. 1), and it is easy to check that the thermodynamic limit of the one-particle density is a periodic function (Corollary 1).

The proof of these results relies on the representation of the modified wave function as a sum over monomer-dimer partitions (Lemma 1), leading to a recurrence relation of second order for the normalization constants, see Eq. (16).

The derivation of the modified wave function starts from the well known representation of Laughlin's wave function as the $p^{\text {th }}$ power of a Vandermonde determinant D, FGIL times a Gaussian weight. The Gaussians can be absorbed into the determinant and we can complete the squares. For $N=2$ particles and $p=3$, this gives

$$
\begin{aligned}
& \left(\exp \left(\gamma z_{2}\right)-\exp \left(\gamma z_{1}\right)\right)^{3} \exp \left(-\frac{1}{2}\left(x_{1}^{2}+x_{2}^{2}\right)\right) \\
& =\operatorname{det}\left(\begin{array}{cc}
\exp \left(-x_{1}^{2} / 6\right) & \exp \left(-x_{2}^{2} / 6\right) \\
\exp \left(\gamma z_{1}-x_{1}^{2} / 6\right) & \exp \left(\gamma z_{2}-x_{2}^{2} / 6\right)
\end{array}\right)^{3} \\
& =\exp \left(\frac{9}{2} \gamma^{2}\right) \operatorname{det}\left(\begin{array}{cc}
\exp \left(-x_{1}^{2} / 6\right) & \exp \left(-x_{2}^{2} / 6\right) \\
\exp \left[\mathrm{i} \gamma y_{1}-\left(x_{1}-3 \gamma\right)^{2} / 6\right] & \exp \left[\mathrm{i} \gamma y_{2}-\left(x_{2}-3 \gamma\right)^{2} / 6\right]
\end{array}\right)^{3} .
\end{aligned}
$$


More generally, with a suitable choice of $\kappa_{N}$ (see Eq. (17) below), Laughlin's function becomes

$$
\Psi_{N}\left(z_{1}, . ., z_{N}\right):=\frac{1}{\sqrt{N !}}\left(\operatorname{det}\left(\varphi_{k-1}\left(z_{j}\right)\right)_{1 \leq k, j \leq N}\right)^{p}
$$

where

$$
\varphi_{k}(z)=\frac{1}{\left(2 \pi \gamma^{-1} \sqrt{\pi}\right)^{1 / 2 p}} \exp (\mathrm{i} k \gamma y) \exp \left(-(x-p k \gamma)^{2} / 2 p\right) .
$$

Notice that, up to the multiplicative constant, the quantity $\gamma$ appears only in combination with the integer $k$. Hence it suffices to consider the case $\gamma=1$. The $\gamma$-dependence can be restored by replacing in the forthcoming formulas integers $k \in \mathbb{Z}$ by $k \gamma$. To simplify the formulas further, we will limit ourselves to $p=3$.

Now we replace the Gaussian in formula (4) by an even measurable function $f$ of compact support. Let

$$
\phi_{k}(z):=\exp (\mathrm{i} k y) f(x-3 k), \quad(k \in \mathbb{Z})
$$

For $N \in \mathbb{N}$, we define the modified wave function $\Phi_{N}$ by

$$
\Phi_{N}\left(z_{1}, . ., z_{N}\right):=\frac{1}{\sqrt{N !}}\left(\operatorname{det}\left(\phi_{k-1}\left(z_{j}\right)\right)_{1 \leq k, j \leq N}\right)^{3} .
$$

It turns out that the one-particle density can be computed exactly when only neighbors overlap: $\phi_{k} \phi_{m}=0$ if $|k-m| \geq 2$. This can be achieved by choosing a function $f$ with a compact support contained in $[-3,3]$, so that

$$
f(\cdot) f(\cdot-3 n)=0 \text { for }|n| \geq 2 \text {. }
$$

The main idea in this section is to use the nearest neighbor overlap condition to simplify the expansion of the determinant power (6). Because of the third power, the expansion of Eq. (6) can be written as a sum of wedge products 3 of functions $\phi_{k}(z) \phi_{m}(z) \phi_{n}(z)$. The overlap condition restricts $k, m, n$ to $k=m=n, k=m$, $n=k+1$, etc. We define an orthonormal set of functions $\psi_{m}$, labelled by their $y$ momentum $m \in \mathbb{Z}$, by:

$$
\begin{aligned}
\phi_{k}^{3}(z)=\sqrt{\alpha_{1}} \psi_{3 k}(z), & \phi_{k}^{2}(z) \phi_{k+1}(z)=\sqrt{\beta_{2}} \psi_{3 k+1}(z) \\
\phi_{k}(z) \phi_{k+1}^{2}(z) & =\sqrt{\beta_{2}} \psi_{3 k+2}(z),
\end{aligned}
$$

The wave function $\psi_{m}(z)$ describes a particle localized around $x=m$. The constants $\alpha_{1}$ and $\beta_{2}$ are suitable positive normalization constants.

The normalization of $\Phi_{N}$ and the one-particle density are easily expressed in terms of $\alpha_{1}, \alpha_{2}:=9 \beta_{2}^{2}$ and $\psi_{m}$ :

\footnotetext{
${ }^{3}$ We use the normalization $(f \wedge g)\left(z_{1}, z_{2}\right)=\frac{1}{\sqrt{2}}\left(f\left(z_{1}\right) g\left(z_{2}\right)-f\left(z_{2}\right) g\left(z_{1}\right)\right)$.
} 
Proposition 1. Let $f$ satisfy the nearest neighbor overlapping condition (7). Let $\lambda_{ \pm}:=\left(\alpha_{1} \pm \sqrt{\alpha_{1}^{2}+4 \alpha_{2}}\right) / 2$. Let $C_{N}$ be the norm squared of $\Phi_{N}$, considered as a function in $L^{2}\left((\mathbb{R} \times[0,2 \pi])^{N}\right)$, and $\rho_{N}(z)$ the one-particle density. Then

$$
\begin{aligned}
C_{N}= & \frac{\lambda_{+}^{N+1}-\lambda_{-}^{N+1}}{\lambda_{+}-\lambda_{-}} \\
\rho_{N}(z)= & \sum_{k=0}^{N-1} \frac{C_{k} \alpha_{1} C_{N-k-1}}{C_{N}}\left|\psi_{3 k}(z)\right|^{2} \\
& +\sum_{k=0}^{N-2} \frac{C_{k} \alpha_{2} C_{N-k-2}}{C_{N}}\left(\left|\psi_{3 k+1}(z)\right|^{2}+\left|\psi_{3 k+2}(z)\right|^{2}\right) .
\end{aligned}
$$

Before we turn to the proof of the proposition, let us observe that it has the periodicity of the one-particle density in the thermodynamic limit as a simple consequence. To see this, observe that translating a function $\psi_{m}$ by a multiple of 3 along the cylinder axis amounts, up to a phase factor, to increasing the index $m$ by a multiple of 3 :

$$
\exp (\mathrm{i} 3 k y) \psi_{m}(z-3 k)=\psi_{m+3 k}(z) .
$$

The density $\rho_{N}(z)$ of Eq. (9) is a function of $x=\operatorname{Re} z$ alone and vanishes when $x$ is far outside the interval $[0,3 N]$. We are interested in the limit of the density around the middle of this interval. For a given $z$, only finitely many $k$ 's contribute to the sum (9). When $x$ is near the middle of the cylinder, the $k$ 's that contribute are of the order of $N / 2$. But now, because of Eq. (8),

$$
\frac{C_{k} \alpha_{1} C_{N-k-1}}{C_{N}} \sim \frac{1}{\lambda_{+}-\lambda_{-}} \frac{\lambda_{+}^{k+1} \alpha_{1} \lambda_{+}^{N-k}}{\lambda_{+}^{N+1}}=\frac{\alpha_{1}}{\lambda_{+}-\lambda_{-}}
$$

when $k$ and $N-k$ both go to infinity. A similar statement holds for the coefficient in the second line of Eq. (9). Hence in the limit $N \rightarrow \infty$, the shifted density $\rho_{N}(z-3\lfloor N / 2\rfloor)$ converges to

$$
\sum_{k=-\infty}^{\infty}\left(\frac{\alpha_{1}}{\lambda_{+}-\lambda_{-}}\left|\psi_{3 k}(z)\right|^{2}+\frac{\alpha_{2} \lambda_{+}^{-1}}{\lambda_{+}-\lambda_{-}}\left(\left|\psi_{3 k+1}(z)\right|^{2}+\left|\psi_{3 k+2}(z)\right|^{2}\right)\right) .
$$

Because of the covariance (10), this function is 3-periodic. Thus we have obtained the following corollary from Prop. [1]

Corollary 1. There is a 3-periodic function $\rho(x)$ of the coordinate $x$ alone such that

$$
\lim _{N \rightarrow \infty} \rho_{N}(z-3\lfloor N / 2\rfloor)=\rho(x)
$$

for all $z=x+\mathrm{i} y \in \mathbb{C}$.

Remark. The corollary does not exclude periods smaller than 3 . The density can even be constant. For example, when $f$ is a step function taking only two values - one on the intersection of $\operatorname{supp} f$ and the shifted supports $\operatorname{supp} f(\cdot-3 n)$ and another one on the remainder of $\operatorname{supp} f$ - a suitable choice of the two values gives a constant density. However, for most choices, the density will have a non-trivial periodicity. 
Now let us turn to the proof of Prop. 1 The proof of the proposition rests on a particular representation of $\Phi_{N}$ in terms of "monomer" and "dimer" functions

$$
\begin{aligned}
u_{\{k\}}(z) & :=\sqrt{\alpha_{1}} \psi_{3 k}(z), \\
u_{\{k, k+1\}}\left(z_{1}, z_{2}\right) & :=-\sqrt{\alpha_{2}}\left(\psi_{3 k+1} \wedge \psi_{3 k+2}\right)\left(z_{1}, z_{2}\right)
\end{aligned}
$$

where $k \in \mathbb{Z}$. In the following lemma, the modified wave function $\Phi_{N}$ is written as a sum over partitions $X_{1}, . ., X_{D}$ of $\{0, \ldots, N-1\}$ into monomers $\{k\}$ and dimers $\{k, k+1\}$. We will always assume that the partitions are ordered, i.e., the elements of $X_{1}$ are smaller than those of $X_{2}$, etc.

Lemma 1. The modified wave function $\Phi_{N}$ can be represented as a sum over ordered monomer-dimer partitions of $\{0, . ., N-1\}$ :

$$
\Phi_{N}=\sum_{\left(X_{1}, . ., X_{D}\right)} u_{X_{1}} \wedge . \wedge u_{X_{D}} .
$$

Proof. The function $\Phi_{N}$ is defined as the power of a determinant. This leads to the expression

$$
\Phi_{N}\left(z_{1}, . ., z_{N}\right)=\frac{1}{\sqrt{N !}}\left(\sum_{\pi} \operatorname{sgn}(\pi) \phi_{\pi(0)}\left(z_{1}\right) \ldots \phi_{\pi(N-1)}\left(z_{N}\right)\right)^{3} .
$$

The sum is over permutations of $\{0, \ldots, N-1\}$. Expanding the power of the sum, we see that $\Phi_{N}$ equals $\sqrt{N !}$ times the antisymmetrization of

$$
\sum_{\sigma, \tau} \operatorname{sgn}(\sigma \tau) \prod_{j=1}^{N} \phi_{j-1}\left(z_{j}\right) \phi_{\sigma(j-1)}\left(z_{j}\right) \phi_{\tau(j-1)}\left(z_{j}\right) .
$$

Suppose $\sigma, \tau$ give a non-vanishing contribution to the sum above. Then because of the nearest neighbor overlapping condition, they must satisfy

$$
\forall k \in\{0, . ., N-1\}:|\sigma(k)-k| \leq 1,|\tau(k)-k| \leq 1,|\tau(k)-\sigma(k)| \leq 1 .
$$

As a consequence, $\sigma$ and $\tau$ are products of disjoint nearest neighbor transpositions $T_{1}, . ., T_{r}$. The set of transpositions is uniquely determined by the permutations $\sigma, \tau$. It can be represented by a monomer-dimer partition of $\{0, \ldots, N-1\}$, with $r$ dimers corresponding to the transpositions' supports. Thus to each pair of permutations fulfilling Eq. (14) we can assign a monomer-dimer partition.

The proof of Eq. (12) is concluded by the following observation: if in Eq. (13) we sum over permutations $\sigma, \tau$ that give the same partition $X_{1}, \ldots, X_{D}$, and then antisymmetrize the resulting sum, we obtain $u_{X_{1}} \wedge . . \wedge u_{X_{D}}$.

To see this, we consider the example $N=3, X_{1}=\{0\}, X_{2}=\{1,2\}$ and leave the general case to the reader. There are three permutation pairs $(\sigma, \tau)$ giving rise to the partition $X_{1}, X_{2}$, namely all combinations of the identity 1 and the transposition (1 2) except $\sigma=\tau=\mathbf{1}$. Their contribution to the sum (13) is

$$
\alpha_{1}^{1 / 2} \beta_{2} \psi_{0}\left(z_{1}\right)\left[-2 \psi_{4}\left(z_{2}\right) \psi_{5}\left(z_{3}\right)+\psi_{5}\left(z_{2}\right) \psi_{4}\left(z_{3}\right)\right]
$$

Antisymmetrization and multiplication by $\sqrt{3 !}$ gives $u_{\{0\}} \wedge u_{\{1,2\}}$. 
Lemma 1 1 allows us to prove Prop. 1 using the orthogonality of contributions from different monomer-dimer partitions.

Proof of Prop. 11. The key observation is that each monomer-dimer partition $X_{1}, . ., X_{D}$ is associated with a unique set of $y$-momenta $m_{1}, . ., m_{N}$ : by the definition (11) of $u_{X}$, we have

$$
u_{X_{1}} \wedge . . \wedge u_{X_{D}}=(-1)^{N-D}\left(\alpha_{N\left(X_{1}\right)} \cdot . . \cdot \alpha_{N\left(X_{D}\right)}\right)^{1 / 2} \psi_{m_{1}} \wedge . . \wedge \psi_{m_{N}} .
$$

The $y$ momenta $\boldsymbol{m}=\left(m_{1}, . ., m_{N}\right)$ are obtained as follows: when $X_{1}, . ., X_{D}$ is a monomer partition, $\boldsymbol{m}$ equals $(0,3, . ., 3 N-3)$. The vector belonging to a partition containing the dimer $\{k, k+1\}$ is obtained from this reference vector by replacing $3 k, 3 k+3$ with $3 k+1,3 k+2$, see Fig. 2. Thus different partitions

a)
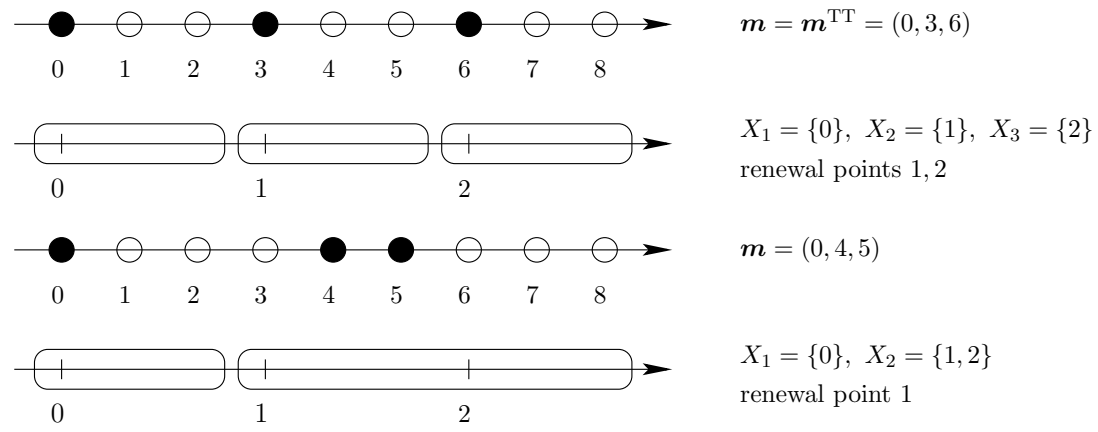

Fig. 2. From partitions of $\{0, \ldots, N-1\}$ to wedge products $\psi_{m_{1}} \wedge \ldots \wedge \psi_{m_{N}}$ : examples for $N=3$. a) A monomer partition corresponds to the Tao-Thouless configuration. b) When a dimer $\{k, k+1\}$ occurs, $3 k, 3 k+3$ is replaced with $3 k+1,3 k+2$ : here, $k=1$. The notion of renewal points is introduced in Def. 1 below. They correspond to starting points of rods, 0 is exluded.

give rise to different sets of $y$-momenta. whence the orthogonality of the various contributions in Eq. (12). The normalization becomes

$$
C_{N}=\left\|\Phi_{N}\right\|^{2}=\sum_{\substack{n_{1}, . ., n_{D} \in\{1,2\}: \\ n_{1}+. .+n_{D}=N}} \alpha_{n_{1}} \cdot . . \cdot \alpha_{n_{D}} .
$$

As a consequence, $C_{N}$ satisfies the recurrence relation

$$
C_{N}=\alpha_{1} C_{N-1}+\alpha_{2} C_{N-2}
$$

with the initial conditions $C_{0}:=1, C_{1}=\alpha_{1}$, whence Eq. (8). Eqs. (12) and (15) lead to

$$
\rho_{N}(z)=C_{N}^{-1} \sum_{X_{1}, . ., X_{D}} \alpha_{N\left(X_{1}\right)} \cdot \ldots \cdot \alpha_{N\left(X_{D}\right)} \sum_{k=1}^{D} v_{X_{k}}(z)
$$

with $v_{\{k\}}(z)=\left|\psi_{3 k}(z)\right|^{2}$ and $v_{\{k, k+1\}}(z)=\left|\psi_{3 k+1}(z)\right|^{2}+\left|\psi_{3 k+2}(z)\right|^{2}$. Changing the order of summation and combining with Eq. (2) gives Eq. (9). 
Remarks. 1. From Eq. (2) we see that $C_{N}$ is a monomer-dimer-partition function for a linear chain of length $N$. The recurrence relation (16) was already given in HL.

2. Similar results hold for $p \neq 3$ and slightly different definitions of monomer and dimer functions.

3. If we relax from the nearest neighbor overlapping condition (7), Lemma 1 still holds true provided we allow polymers of arbitrary length instead of only monomers and dimers. The normalization constants then satisfy a recurrence relation of infinite order.

4. One last remark concerns the comparison with the torus wave functions introduced by Haldane and Rezayi $[\mathrm{HR}$. We can define the modified torus wave function $\tilde{\Phi}_{N}$ by Eq. (6), replacing the functions $\phi_{k}$ on the right-hand side by periodified functions

$$
\tilde{\phi}_{k}(z):=\sum_{n=-\infty}^{\infty} \exp (\mathrm{i} n N y) \phi_{k}(z-3 N n)=\sum_{n=-\infty}^{\infty} \phi_{k+n N} .
$$

If in Eq. (51) $\phi_{k}$ is defined with Gaussians $f(x)=\exp \left(-x^{2} / 2 p\right)$ instead of a function with compact supports, the modified function $\tilde{\Phi}_{N}$ is a Haldane-Rezayi torus function. When the nearest neighbor overlapping condition is satisfied, the torus function $\tilde{\Phi}_{N}$ has an explicitly computable normalization and one-particle density. It is associated with a monomer-dimer system on a ring, with one long additional polymer covering the whole ring. In the limit of long tori, we recover the density of the cylinder modified function. Thus for the solvable model, one can check that torus and cylinder functions are equivalent.

To summarize, we have seen how the representation (12) of $\Phi_{N}$ in terms of a "quantum monomer-dimer" system leads to simple formulas for the normalization constants and the one-particle density; the periodicity of the one-particle density in the thermodynamic limits stems from the translational invariance of the corresponding monomer-dimer system.

In the next section, we will see that Laughlin's wave function admits a similar representation in terms of a polymer system, and the mechanism leading to a periodic one-particle density is essentially the same. However, the situation is complicated by the existence of polymers of arbitrary length and non-vanishing activity.

\section{Laughlin's cylinder function}

This section is devoted to the proof of the main results of this paper. In the first subsection, we prove general properties of Laughlin's function. We proceed with a polymer representation (Subsect. 3.2), leading to a recurrence relation of infinite order for the normalization constants. This relation is exploited in Subsect. 3.3 where we prove an important technical result on the asymptotics of normalization constants. The existence of the thermodynamic limit of Laughlin's state and its periodicity are shown in Subsect. 3.4. Symmetry breaking and clustering properties are proved in Subsect. 3.5.

Throughout this section, we will assume that $p$ is odd. However, most results hold for even $p$, with minor modifications (e.g., replacing wedge products with 
symmetric tensor products). The multiplicative constant in the definition (3) of $\Psi_{N}$ will be fixed as

$$
\kappa_{N}=\frac{1}{\sqrt{N !}} \frac{1}{\left(2 \pi \gamma^{-1} \sqrt{\pi}\right)^{N / 2}} \exp \left(-\frac{1}{2} p^{2} \gamma^{2} \sum_{j=0}^{N-1} j^{2}\right)
$$

3.1. Basic properties. Laughlin's wave function has a few number of simple, but important properties that we present in this subsection. They allow us to derive symmetries of Laughlin's state and to show that in the limit of infinitely many particles, Laughlin's state cannot give rise to a constant density. This holds regardless of the size of the radius, in contrast with the results proved in Subsects. 3.4 and 3.5 .

All properties rely on the expansion of $\Psi_{N}$ as a sum of wedge products of lowest Landau level basis functions

$$
\psi_{k}(z):=\frac{1}{\sqrt{2 \pi \gamma^{-1} \sqrt{\pi}}} \exp (\mathrm{i} k \gamma y) \exp \left(-\frac{1}{2}(x-k \gamma)^{2}\right), \quad k \in \mathbb{Z} .
$$

They form an orthonormal set in $L^{2}\left(\mathbb{R} \times\left[0,2 \pi \gamma^{-1}\right]\right)$. One basis function can be transformed into another by a shift in the axial direction:

$$
t\left(\gamma \boldsymbol{e}_{\mathrm{x}}\right) \psi_{k}=\psi_{k+1} \quad(k \in \mathbb{Z})
$$

where $t\left(\gamma \boldsymbol{e}_{\mathrm{x}}\right)$ is the magnetic translation $\psi(z) \rightarrow \exp (1 \gamma y) \psi(z-\gamma)$.

The expansion of $\Psi_{N}$ can be obtained from the expansion of the $p$-th power of the Vandermonde determinant into monomials. Define coefficients $a_{N}(\boldsymbol{m})$ and $b_{N}(\boldsymbol{m})$ by

$$
\begin{aligned}
\Psi_{N} & =\sum_{0 \leq m_{1}<. .<m_{N} \leq p N-p} a_{N}\left(m_{1}, . ., m_{N}\right) \psi_{m_{1}} \wedge . \wedge \psi_{m_{N}} \\
\prod_{1 \leq j<k \leq N}\left(z_{k}-z_{j}\right)^{p} & =\sum_{0 \leq m_{1}, . ., m_{N} \leq p N-p} b_{N}\left(m_{1}, \ldots, m_{N}\right) z_{1}^{m_{1}} \ldots z_{N}^{m_{N}} .
\end{aligned}
$$

A straight-forward computation then gives

$$
a_{N}\left(m_{1}, . ., m_{N}\right)=b_{N}\left(m_{1}, . ., m_{N}\right) \exp \left(\frac{1}{2} \gamma^{2} \sum_{j=1}^{N}\left(m_{j}^{2}-p^{2}(j-1)^{2}\right)\right) \text {. }
$$

This equation allows us to translate properties of the $p$-th power of the Vandermonde determinant into properties of $\Psi_{N}$.

The power of the Vandermonde determinant (19) is a homogeneous polynomial of total degree $p N(N-1) / 2$. Therefore $\Psi_{N}$ has definite $y$-momentum

$$
-\mathrm{i} \sum_{k=1}^{N} \frac{\partial}{\partial y_{k}} \Psi_{N}=\frac{p N(N-1) \gamma}{2} \Psi_{N}
$$

As a consequence, the one-particle density will be independent of the angular coordinate $y$. An additional symmetry comes from the "reversal invariance" D, 
FGIL $b_{N}\left(m_{1}, . ., m_{N}\right)=b_{N}\left(p N-p-m_{N}, . ., p N-p-m_{1}\right):$ Laughlin's state is invariant with respect to a $180^{\circ}$ rotation around the middle of the cylinder,

$$
s_{p(N-1) \gamma / 2}^{\otimes N} \Psi_{N}=\Psi_{N}
$$

Here $s_{a}$ refers to a "magnetic" rotation, $\psi(z) \rightarrow \exp (\mathrm{i} 2 a y) \psi(2 a-z)$. Notice that $s_{r \gamma / 2} \psi_{k}=\psi_{2 r-k}$.

Because of Eqs. (18) and (21), the one-particle density can be expressed as:

$$
\rho_{N}(z)=\sum_{k=0}^{p N-p}\left\langle c_{k}^{*} c_{k}\right\rangle_{N}\left|\psi_{k}(z)\right|^{2} \propto \sum_{k=0}^{p N-p}\left\langle c_{k}^{*} c_{k}\right\rangle_{N} \exp \left(-(x-k \gamma)^{2}\right) .
$$

$\langle\cdot\rangle_{N}$ refers to expectation values in the state $\Psi_{N} /\left\|\Psi_{N}\right\|$ and $c_{k}^{*}, c_{k}$ are the fermionic creation and annihilation operators for the state $\psi_{k}$, e.g., $c_{k}^{*} f=$ $\psi_{k} \wedge f$. Notice that the expectation values $\left\langle c_{k}^{*} c_{m}\right\rangle, k \neq m$, vanish due to the $y$-invariance (21).

We are interested in limits of $\rho_{N}(z-p\lfloor N / 2\rfloor \gamma)$ as $N \rightarrow \infty$. Eq. (23) shows that any limit point of the shifted density is a sum of Gaussians. The following lemma deduces several statements from this observation.

Lemma 2. Let $\left(n_{k}\right)_{k \in \mathbb{Z}}$ be a sequence of numbers in $[0,1]$ that does not identically vanish, and $\rho(x):=\sum_{k=-\infty}^{\infty} n_{k}\left|\psi_{k}(x)\right|^{2}$. Then

1. $\rho(x)$ cannot be a constant.

2. If $\rho(x)$ is periodic, every period must be a multiple of $\gamma$.

3. $\rho(x)$ is periodic with period $p \gamma$ if and only if $\left(n_{k}\right)$ is periodic with period $p$.

Proof. $\rho=f * \mu$ is the convolution of the function $f(x):=\left(2 \pi \gamma^{-1} \sqrt{\pi}\right)^{-1} \exp \left(-x^{2}\right)$ and the measure $\mu=\sum_{k \in \mathbb{Z}} n_{k} \delta_{k \gamma}$. With $\rho$ and $\mu$ we can associate tempered distributions $T_{\rho}$ and $T_{\mu}$ :

$$
T_{\rho} \phi:=\int_{-\infty}^{\infty} \rho(x) \phi(x) \mathrm{d} x, \quad T_{\mu} \phi:=\int_{\mathbb{R}} \phi \mathrm{d} \mu .
$$

Their Fourier transforms $(\hat{T} \phi:=T \check{\phi})$ satisfy

$$
\hat{T}_{\rho}=\sqrt{2 \pi} \hat{f} \hat{T}_{\mu}, \quad \hat{f}(k)=\frac{\gamma}{2 \pi} \exp \left(-k^{2} / 4\right)
$$

The product $\hat{f} \hat{T}_{\mu}$ is the distribution $\left(\hat{f} \hat{T}_{\mu}\right) \phi:=\hat{T}_{\mu}(\hat{f} \phi)$. It follows from Eq. (24) that the mapping $\mu \mapsto f * \mu$ is injective. Therefore $f$ and $\mu$ must have the same periodicity. Since any period of $\mu$ is obviously a multiple of $\gamma$, the same must be true for periods of $\rho$. This excludes a constant density, since constant functions admit periods that are not multiples of $\gamma$.

Notice that it is not completely trivial that the density cannot be constant. If instead of Gaussians we added up more general functions, we could obtain a constant. Examples include functions of compact support, see the remark on p. 8 and log-concave functions, as was shown in [BL], Sect. 1.4.3. 
Observe also that there is a relationship not only between the periods of $\left(n_{k}\right)$ and $\rho(x)$, but also between the amplitudes of oscillation. If $\rho(x)$ is $p \gamma$-periodic, the $k$-th Fourier coefficients can be expressed in terms of occupation numbers:

$$
\frac{1}{p \gamma} \int_{0}^{p \gamma} \rho(x) \exp \left(-\mathrm{i} \frac{2 \pi k x}{p \gamma}\right) \mathrm{d} x=\frac{1}{p} \frac{1}{2 \pi} \exp \left(-\frac{\pi^{2} k^{2}}{p^{2} \gamma^{2}}\right) \sum_{j=0}^{p-1} n_{j} \exp \left(-\mathrm{i} \frac{2 \pi k j}{p}\right)
$$

This relation generalizes Poisson's summation formula, which shows that the Fourier coefficients of $\rho(x)$ in the filled Landau level $\left(p=1\right.$ and $\left.n_{k} \equiv 1\right)$ are $(2 \pi)^{-1} \exp \left(-\pi^{2} k^{2} / \gamma^{2}\right)$.

3.2. Associated polymer system and renewal equation. In this subsection we show that Laughlin's wave function admits a representation as a sum over certain partitions of $\{0, . ., N-1\}$, analogous to Lemma 1 for the solvable model. Instead of only monomers or dimers, the partition may contain longer polymers or "rods" $X=\{j, . ., j+n-1\}$. The normalization becomes a discrete polymer partition function GK with translationally invariant activity and satisfies a discrete renewal equation, as explained at the end of this subsection.

Let $N(X)$ denote the cardinality or "length" of a $\operatorname{rod} X$, and let $\mathcal{P}_{N}$ be the set of ordered partitions $X_{1}, . ., X_{D}$ of $\{0,1, . ., N-1\}$ into rods $X_{j}$. The following holds:

Proposition 2 (Associated polymer system). There is a mapping associating with each rod $X$ an antisymmetric function $u_{X}$ of $N(X)$ complex variables so that

$$
\begin{gathered}
\Psi_{N}=\sum_{\left(X_{1}, . ., X_{D}\right) \in \mathcal{P}_{N}} u_{X_{1}} \wedge . \wedge u_{X_{D}}, \\
C_{N}:=\left\|\Psi_{N}\right\|^{2}=\sum_{\left(X_{1}, . ., X_{D}\right) \in \mathcal{P}_{N}} \Phi\left(X_{1}\right) \cdot . . \cdot \Phi\left(X_{D}\right), \quad \Phi(X)=\left\|u_{X}\right\|^{2} .
\end{gathered}
$$

The expansion (25) for $N=3$ particles and $p=3$ is explicitly written down in JLS, Eq. (10), up to a small difference: in JLS, the sum is over partitions of the set $\{-1, \ldots, 3 N-2\}$ instead of $\{0, \ldots, N-1\}$, and rods $X$ have length $|X|=3,6, \ldots$ instead of $|X|=1,2, \ldots$ as is the case here.

We will see that the family of "polymer functions" $\left(u_{X}\right)$ has two important additional properties. The first is a kind of localization: $u_{X}$ is a sum of wedge products of functions $\psi_{k}$ with indices $k$ in $\{p \min X, \ldots, p \max X-p\}$. The second property is translational covariance:

$$
\forall j \in \mathbb{Z}, \quad u_{j+X}=t\left(j p \gamma \boldsymbol{e}_{\mathrm{x}}\right)^{\otimes N(X)} u_{X}
$$

Thus the shift of a rod results in the magnetic translation of the corresponding function. This covariance is at the origin of $p \gamma$-periodicity in Laughlin's state.

Eq. (26) says that the normalization $C_{N}$ is a polymer partition function with activity $\Phi(X)$. As a consequence of the covariance (27), the activity is translationally invariant, i.e., the activity of a rod depends on its length $N(X)$ only: 
we can define non-negative numbers $\left(\alpha_{n}\right)_{n \in \mathbb{N}}$ by

$$
\Phi(X)=\left\|u_{X}\right\|^{2}=\alpha_{N(X)} .
$$

The numbers $\left(\alpha_{n}\right)$ will appear as coefficients in a recurrence relation for the normalization $C_{N}$.

The proof of the previous proposition makes crucial use of a product rule proved in FGIL for the expansion coefficients $b_{N}(\boldsymbol{m})$. It is more easily expressed with some auxiliary definitions.

Definition 1. Let $\boldsymbol{m}=\left(m_{1}, . ., m_{N}\right) \in \mathbb{Z}^{N}$ with $m_{1} \leq . . \leq m_{N}$.

$-\boldsymbol{m}$ is $N$-admissible if

$$
\sum_{j=1}^{k} m_{j} \geq \sum_{j=1}^{k} p(j-1)
$$

for all $k \in\{1, \ldots, N\}$ with equality for $k=N$.

$-k \in\{1, \ldots, N-1\}$ is a renewal point of $\boldsymbol{m}$ if

$$
\sum_{j=1}^{k} m_{j}=\sum_{j=1}^{k} p(j-1) .
$$

- $\boldsymbol{m}$ is reducible if it admits a renewal point $k \in\{1, \ldots, N-1\}$, and irreducible in the opposite case.

Note that $N$-admissibility actually implies $0 \leq m_{1}, . ., m_{N} \leq p N-p$. The reducibility of a sequence is most easily visualized with the help of the sequence of occupation numbers in Laughlins' state, see JLS, Sect. 4.1.

There are various equivalent characterizations of $N$-admissibility [FGIL, RH, KTW]. In all of them, the so-called Tao-Thouless configuration TT.

$$
\boldsymbol{m}^{\mathrm{TT}}:=(0, p, 2 p, . ., p N-p)
$$

plays the role of a reference configuration. In the proof of the next lemma, we will use the following: a vector $\boldsymbol{m}$ is $N$-admissible if and only if it is majorized by $\boldsymbol{m}^{\mathrm{TT}}$, which is equivalent to the existence of a doubly stochastic matrix $P$ such that

$$
\boldsymbol{m}=P \boldsymbol{m}^{\mathrm{TT}}
$$

see e.g. HLP, p. 49.

This characterization allows us to visualize renewal points as block-diagonal matrices: If $\boldsymbol{m}$ is given by Eq, (30) for some block-diagonal, doubly stochastic matrix $P$ that has an upper left $k \times k$ block, $k$ is a renewal point of $\boldsymbol{m}$. Conversely, if $k$ is a renewal point of the $N$-admissible vector $\boldsymbol{m}$, every doubly stochastic matrix fulfilling Eq. (30) must be block-diagonal.

By the Birkhoff-von Neumann theorem, a matrix is doubly stochastic if and only if it is a convex combination of permutation matrices. Interestingly, the

\footnotetext{
4 Here, we use that $\|\cdot\|$ refers to integration over the infinite cylinder $\mathbb{R} \times\left[0,2 \pi \gamma^{-1}\right]$. On finite cylinders, the activity is not completely translationally invariant due to boundary effects, although Prop. 2 and the covariance (27) stay true.
} 
only matrices that we shall need are equally weighted averages of permutation matrices, see Eq. (34) below.

The following lemma contains fundamental properties of the expansion coefficients $a_{N}(\boldsymbol{m})$ :

Lemma 3. Let $\boldsymbol{m}=\left(m_{1}, . ., m_{N}\right)$ with $m_{1} \leq . . \leq m_{N}$.

1. Suppose $a_{N}\left(m_{1}, . ., m_{N}\right) \neq 0$. Then $\boldsymbol{m}$ is $N$-admissible.

2. Suppose $k \in\{1, \ldots, N\}$ is a renewal point of $\boldsymbol{m}$. Then:

(a) $\boldsymbol{m}$ is $N$-admissible if and only if $\left(m_{1}, . ., m_{k}\right)$ and $\left(m_{k}-p k, \ldots, m_{N}-p k\right)$ are $k$-resp. $(N-k)$-admissible.

(b) The following product rule holds:

$$
a_{N}\left(m_{1}, . ., m_{N}\right)=a_{k}\left(m_{1}, . ., m_{k}\right) a_{N-k}\left(m_{k+1}-p k, . ., m_{N}-p k\right) .
$$

We will frequently write Eq. (31) as

$$
a_{N}(\boldsymbol{m})=a_{N}\left(\boldsymbol{m}_{1}^{N}\right)=a_{k}\left(\boldsymbol{m}_{1}^{k}\right) a_{N-k}\left(\boldsymbol{m}_{k+1}^{N}-p k\right)
$$

using the short-hands $\boldsymbol{m}_{a}^{b}:=\left(m_{a}, . ., m_{b}\right)$ and $\left(x_{1}-b, . ., x_{n}-b\right)=: \boldsymbol{x}-b$.

Proof. The previous lemma holds true if $a_{N}$ is replaced with the coefficients $b_{N}$ defined in Eq. (19). The corresponding statements for $b_{N}$ have been proved in FGIL, and the statements for $a_{N}$ may be deduced with the help of Eq. (20).

The proof given in FGIL uses the polynomial character of the Vandermonde determinant. However, in view of the results on the solvable model (Sect. 2), we should like to stress the importance of the fact that we have the power of a determinant. Therefore we give a new proof. For the sake of simplicity, we take $p=3$; the proof for other values of $p$ is similar.

1. Expanding the right-hand side of

$$
\prod_{1 \leq j<k \leq N}\left(z_{k}-z_{j}\right)^{3}=\left(\sum_{\pi \in \mathcal{S}_{N}} \operatorname{sgn}(\pi) z_{1}^{\pi(1)-1} \ldots z_{N}^{\pi(N)-1}\right)^{3}
$$

we find

$$
b_{N}\left(m_{1}, . ., m_{N}\right)=\sum_{\substack{\pi, \sigma, \tau \in \mathcal{S}_{N}: \\ m=\pi+\sigma+\tau-3}} \operatorname{sgn}(\pi \sigma \tau),
$$

where the short-hand $\boldsymbol{m}=\pi+\sigma+\tau-3$ in the subscript stands for

$$
\forall j \in\{1, . ., N\}: m_{j}=\pi(j)+\sigma(j)+\tau(j)-3 .
$$

Now suppose $b_{N}(\boldsymbol{m}) \neq 0$. Then there exist permutations $\pi, \sigma, \tau$ adding up to $\boldsymbol{m}$ in the sense of the previous equation. In terms of the associated permutation matrices,

$$
\boldsymbol{m}=P \boldsymbol{m}^{\mathrm{TT}}, \quad P=\frac{1}{3}\left(P_{\pi}+P_{\sigma}+P_{\tau}\right)
$$

where $\boldsymbol{m}^{\mathrm{TT}}$ is as in Eq. (29) with $p=3 . P$ is doubly stochastic and therefore $\boldsymbol{m}$ is $N$-admissible. Since by Eq. (20) $a_{N}(\boldsymbol{m}) \neq 0$ if and only if $b_{N}(\boldsymbol{m}) \neq 0$, the first statement of the lemma follows. 
2. Let $\boldsymbol{m}=\left(m_{1}, \ldots, m_{N}\right)$ with $m_{1} \leq . . \leq m_{N}$. and let $k$ be a renewal point of $\boldsymbol{m}$. 2.(a) is proven in $\mathrm{KTW}$. For 2.(b), suppose $\pi, \sigma, \tau$ are permutations such that Eq. (34) holds. Then the matrix $P$ from this equation must be blockdiagonal with an upper left $k \times k$ block. This means that $\pi, \sigma, \tau$ must leave $\{1, . ., k\}$ and $\{k+1, . ., N\}$ invariant. As a consequence, the sum (33) factorizes into two sums

$$
b_{N}(\boldsymbol{m})=\left(\sum_{\pi^{\prime}, \sigma^{\prime}, \tau^{\prime}}^{\prime} \operatorname{sgn}\left(\pi^{\prime} \sigma^{\prime} \tau^{\prime}\right)\right)\left(\sum_{\pi^{\prime \prime}, \sigma^{\prime \prime}, \tau^{\prime \prime}}^{\prime \prime} \operatorname{sgn}\left(\pi^{\prime \prime} \sigma^{\prime \prime} \tau^{\prime \prime}\right)\right) .
$$

The first sum is over permutations of $\{1, . ., k\}$ such that

$$
\boldsymbol{m}_{1}^{k}=\pi^{\prime}+\sigma^{\prime}+\tau^{\prime}-3,
$$

and equals $b_{k}\left(m_{1}, . ., m_{k}\right)$. The second sum is over permutations of $\{k+1, . ., N\}$ such that

$$
\boldsymbol{m}_{k+1}^{N}=\pi^{\prime \prime}+\sigma^{\prime \prime}+\tau^{\prime \prime}-3
$$

Rewriting this equation with permutations of $\{1, . ., N-k\}$ instead of $\{k+$ $1, . ., N\}$, one finds that the second sum equals $b_{N-k}\left(\boldsymbol{m}_{k+1}^{N}-3 k\right)$. The product rule thus holds for $b_{N}(\boldsymbol{m})$. A straight-forward computation on the exponential factor in (20) allows us to conclude that $a_{N}(\boldsymbol{m})$ satisfies the product rule too.

Now we are able to prove Prop. 2

Proof of Prop. Q In the expansion (18), we group together admissible $\boldsymbol{m}$ 's that have the same set of renewal points $r_{1}<. .<r_{D}$. The product rule (31) gives

$$
\begin{aligned}
a_{N}(\boldsymbol{m}) \psi_{m_{1}} \wedge . . \wedge \psi_{m_{N}} & \\
=\left(a_{r_{1}}\left(\boldsymbol{m}_{1}^{r_{1}}\right) \psi_{m_{1}} \wedge .\right. & \left.\wedge \psi_{m_{r_{1}}}\right) \wedge\left(a_{r_{2}-r_{1}}\left(\boldsymbol{m}_{r_{1}+1}^{r_{2}}-p r_{1}\right) \psi_{m_{r_{1}+1}} \wedge . \wedge \psi_{m_{r_{2}}}\right) \\
& \wedge . . \wedge\left(a_{N-r_{D}}\left(\boldsymbol{m}_{r_{D}+1}^{N}-p r_{D}\right) \psi_{m_{r_{D}+1}} \wedge . . \wedge \psi_{m_{N}}\right) .
\end{aligned}
$$

The renewal points split $\boldsymbol{m}$ into $D+1$ irreducible blocks. This motivates the definition of polymer functions $u_{X}$ as sums over irreducible sequences:

$$
u_{\{a, . ., a+n-1\}}:=\sum_{\substack{m \text { m-admissible } \\ \text { and irreducible }}} a_{n}(\boldsymbol{m}) \psi_{m_{1}+p a} \wedge . . \wedge \psi_{m_{n}+p a} .
$$

These functions fulfill the covariance (27). Combining the expansion (18) and Eq. (35), we obtain

$$
\Psi_{N}=\sum_{D=0}^{N-1} \sum_{0<r_{1}<. .<r_{D}<N} u_{\left\{0, . ., r_{1}-1\right\}} \wedge u_{\left\{r_{1}, . ., r_{2}-1\right\}} \wedge . . \wedge u_{\left\{r_{D}, . ., N-1\right\}},
$$

which concludes the proof. 
The results presented in this article rely heavily on the relationship of $\Psi_{N}$ and polymer systems presented in Prop. 2. Let us recall some basic facts on polymer systems with translationally invariant activity supported by $\operatorname{rods}\{j, . ., j+n-1\}$ and the link to renewal equations. In view of Eq. (28), the expression (26) for the polymer partition function $C_{N}$ becomes

$$
C_{N}=\sum_{\substack{D, n_{1}, . ., n_{D} \in \mathbb{N}: \\ n_{1}+. .+n_{D}=N}} \alpha_{n_{1} \ldots \alpha_{n_{D}}}
$$

It follows that $C_{N+M} \geq C_{N} C_{M}$. This supermultiplicativity is a general property of polymer partition functions, see [GK]. As a consequence, we can define

$$
-\ln r:=\sup _{N \in \mathbb{N}} \frac{1}{N} \ln C_{N}=\lim _{N \rightarrow \infty} \frac{1}{N} \ln C_{N},
$$

where we use the convention $-\ln 0=\infty$. The left-hand side $-\ln r$ is the pressure of the polymer system; note that $r$ is the radius of convergence of the power series $\sum_{n} C_{n} t^{n}$. If we suppose in addition that there exists some $\xi>0$ such that the rescaled activity $\xi^{N} \Phi: X \mapsto \xi^{N(X)} \Phi(X)$ is stable, i.e.,

$$
\forall k \in \mathbb{Z}: \sum_{X \ni k} \frac{\xi^{N(X)} \Phi(X)}{N(X)}<\infty,
$$

the pressure $-\ln r$ is finite. This follows from general theorems GK but can be shown here using a particularly simple argument: the stability condition (39) is equivalent to $\sum_{n} \xi^{n} \alpha_{n}<\infty$. It is fulfilled for some $\xi>0$ if and only if the power series $\sum_{n} \alpha_{n} t^{n}$ has a nonvanishing radius of convergence. On the other hand, Eq. (37) leads to the formal power series identity

$$
C(t)=1+\sum_{n=1}^{\infty} C_{n} t^{n}=\frac{1}{1-\sum_{n=1}^{\infty} \alpha_{n} t^{n}}=\frac{1}{1-\alpha(t)}
$$

Therefore, if $\alpha(t)$ has a positive radius of convergence, so has $\sum_{n} C_{n} t^{n}$, whence $r>0$ and $-\ln r<\infty$.

The type of polymer systems envisaged here bears an interesting connection to renewal theory, as observed by IVZ] (see [Fel] for an account on renewal theory). The formal power series identity (40) is equivalent to the recurrence relation

$$
\forall n \in \mathbb{N}: \quad C_{n}=\alpha_{1} C_{n-1}+\ldots+\alpha_{n}, \quad C_{0}:=1
$$

This recurrence relation is known in stochastics as a (discrete) renewal equation. If we interpret $\mathbb{Z}$ as a discrete time axis and starting points of rods as events, the activity $\left(\alpha_{n}\right)$ is related to a probability distribution on waiting times between events, and $C_{n}$ is related to the probability that an event occurs (i.e., that $n$ is a renewal point). More precisely, we suppose that $r>0$ and set $p_{n}:=r^{n} \alpha_{n}$ and $u_{n}:=r^{n} C_{n}$. Suppose that the sequence of activities is aperiodic, i.e.,

$$
\operatorname{gcd}\left\{n \in \mathbb{N} \mid \alpha_{n}>0\right\}=1 .
$$

Then one of the following cases necessarily holds: 
1. $\left(p_{n}\right)$ defines a probability measure on $\mathbb{N}$ with finite mean $\mu$. In this case $u_{n}=r^{n} C_{n} \rightarrow \mu^{-1}>0$. (The associated renewal process is positive recurrent.)

2. $\left(p_{n}\right)$ defines a probability measure on $\mathbb{N}$ with infinite mean $\mu=\infty$. Then $u_{n}=r^{n} C_{n} \rightarrow 0$ (but $\sum_{n} u_{n}=\infty$ ). (The renewal process is null recurrent.)

3. $\left(p_{n}\right)$ defines a defective measure on $\mathbb{N}$ (i.e., $\sum_{n} p_{n}<1$ ). Then $\sum_{n} u_{n}=$ $\left(1-\sum_{n} p_{n}\right)^{-1}<\infty$, whence $u_{n} \rightarrow 0$. (The renewal process is transient.)

See [Fel] for a proof. We will refer to the first case as a renewal process with finite mean. Thus in any case, $r^{n} C_{n} \rightarrow q \geq 0$, but $q>0$ if and only if the associated renewal process has finite mean.

3.3. Large $N$-asymptotics of normalization constants. In this subsection, we exploit the relation of $\Psi_{N}$ with polymer systems and renewal equation to investigate the asymptotics of the normalization constant $C_{N}=\left\|\Psi_{N}\right\|^{2}$. The result comes in two parts: first, for all values of the radius, there exist a $r>0$ and $q \geq 0$ such that $C_{N} r^{N} \rightarrow q \geq 0$ (Lemma 4). Second, for sufficiently large $\gamma$ (thin cylinders), $q$ is strictly positive (Theorem 1). Thus the associated polymer system, suitably rescaled, has a stable activity in the sense of Eq. (39), and on sufficiently thin cylinders, the associated renewal process has finite mean.

In addition, we give lower and upper bounds on $r$ that are interesting in the context of Laughlin's plasma analogy. Our bounds are consistent with results by For on the free energy of a jellium system placed on a cylinder with large radius.

Lemma 4. Let $p \in \mathbb{N}$ and $\gamma>0$. There exist $r>0$ and $q \geq 0$ such that

$$
-\ln r=\lim _{N \rightarrow \infty} \frac{1}{N} \ln C_{N}=\sup _{N} \frac{1}{N} \ln C_{N}, \quad q=\lim _{n \rightarrow \infty} C_{n} r^{n} .
$$

Moreover, $r$ satisfies the bound

$$
\left(e^{p} \sum_{\substack{n_{1}, \ldots, n_{p} \in \mathbb{Z}: \\ n_{1}+\ldots+n_{p}=0}} \exp \left(-\frac{\pi^{2}}{p \gamma^{2}}\left(n_{1}^{2}+. .+n_{p}^{2}\right)\right)\right)^{-1} \leq r p^{1-\frac{p}{2}}\left(\frac{e \gamma}{\sqrt{\pi}}\right)^{1-p} \leq 1
$$

Proof. We know already that $-\ln r=\lim _{n} n^{-1} \log C_{n}$ exists, see (38). The strict positivity of $r$ will follow from Ineq. (43). The observation of the previous subsection yields the existence of $q=\lim _{n} C_{n} r^{n}$; note that $\alpha_{1}=\left\|\psi_{0}\right\|^{2}=1$ so that the sequence of activities $\left(\alpha_{n}\right)$ fulfills the aperiodicity condition (42).

Thus it remains to prove Ineq. (43). The idea is to use the representation (4) of $\Psi_{N}$ as the $p$-th power of a determinant times $1 / \sqrt{N !}$ and to give lower and upper bounds on $C_{N}$ using Hölder's and Hadamard's inequalities. These inequalities have already been used in FGIL, Sect. 3.3., to derive bounds on the free energy of jellium on a sphere. We start with the application of Hadamard's inequality, which gives

$$
\left|\Psi_{N}\left(z_{1}, . ., z_{N}\right)\right|^{2} \leq \frac{1}{N !} \prod_{j=1}^{N}\left(\sum_{k=0}^{N-1}\left|\varphi_{k}\left(z_{j}\right)\right|^{2}\right)^{p}
$$


It follows that

$$
C_{N} \leq \frac{1}{N !}\left(\int_{-\infty}^{\infty} \frac{1}{\sqrt{\pi}}\left(\sum_{k=0}^{N-1} \exp \left(-\frac{(s-p k \gamma)^{2}}{p}\right)\right)^{p} \mathrm{~d} s\right)^{N} .
$$

The integral from $-\infty$ to $-p \gamma / 2$ and from $(N-1 / 2) p \gamma$ to $\infty$ can be bounded by an $N$-independent constant. The integral from $-p \gamma / 2$ to $(N-1 / 2) p \gamma$ is bounded from above by

$$
\frac{1}{\sqrt{\pi}} N \int_{0}^{p \gamma} f(x)^{p} \mathrm{~d} x, \quad f(x):=\sum_{k=-\infty}^{\infty} \exp \left(-\frac{(x-p k \gamma)^{2}}{p}\right) .
$$

Representing $f$ as a Fourier series via Poisson's summation formula, we find that the first expression in (44) equals $N b(\gamma)$ with

$$
b(\gamma)=p^{1-\frac{p}{2}}\left(\frac{\sqrt{\pi}}{\gamma}\right)^{p-1} \sum_{\substack{n_{1}, . ., n_{p} \in \mathbb{Z}: \\ n_{1}+. .+n_{p}=0}} \exp \left(-\frac{\pi^{2}}{p \gamma^{2}}\left(n_{1}^{2}+. .+n_{p}^{2}\right)\right) .
$$

Thus we get $C_{N} \leq(N b(\gamma)+c)^{N} / N$ !, from which the lower bound on $r$ is easily obtained.

Now we turn to a lower bound for $C_{N}$. With Hölder's inequality written as

$$
\int_{\Omega} g^{p} \geq|\Omega|^{p-1}\left|\int_{\Omega} g\right|^{p}
$$

applied to the domain of integration $\left([-p \gamma / 2,(N-1 / 2) p \gamma] \times\left[0,2 \pi \gamma^{-1}\right]\right)^{N}$, we find

$$
C_{N} \geq\left(\frac{N ! \sqrt{\pi}^{N}}{(N p \gamma)^{N}}\right)^{p-1} \sqrt{p}^{p N} \prod_{k=0}^{N-1}\left(1-\epsilon_{N-k-\frac{1}{2}}-\epsilon_{k+\frac{1}{2}}\right)^{p}
$$

where $\epsilon_{m}=[\operatorname{erfc}(m \sqrt{p} \gamma)] / 2$ and erfc is the complementary error function. The product over $k$ does not contribute to $\lim N^{-1} \log C_{N}$. Making use of Stirling's formula, we obtain the desired upper bound to $r$.

Remark. The bounds (43) lead to a statement on the thick cylinder asymptotics of $r: r=O\left(\gamma^{p-1}\right)$ as $\gamma \rightarrow 0$. This complements the thin cylinder $(\gamma \rightarrow \infty)$ asymptotics given in Eq. (48) below.

Lemma 4 leaves open the question whether $q>0$ or $q=0$, i.e., whether the associated renewal process has finite or infinite mean. In order to answer this question, it is useful to have a closer look at the activity $\left(\alpha_{n}\right)$.

Lemma 5 ( $\gamma$-dependence of the activity). Monomers have activity $\alpha_{1}=1$. The activity of a polymer of length $N \geq 2$ is a polynomial of $\exp \left(-\gamma^{2}\right)$ with minimal degree $p(N-1)$ and coefficient in $\mathbb{N}_{0}$. In particular,

$$
\alpha_{N}=O\left(\left(\exp \left(-\gamma^{2}\right)\right)^{p(N-1)}\right) \text { as } \gamma \rightarrow \infty \text {. }
$$

Hence, in the thin cylinder limit, only monomers have a non-vanishing activity. 
Proof. The monomer functions are $u_{\{k\}}(z)=\psi_{p k}(z)$, whence $\alpha_{1}=1$. Combining Eqs. (20) and (36), we find

$$
\alpha_{N}=\sum_{\boldsymbol{m} \text { irreducible }}\left|b_{N}(\boldsymbol{m})\right|^{2}\left(e^{-\gamma^{2}}\right)^{\sum_{j=1}^{N}\left(p^{2}(j-1)^{2}-m_{j}^{2}\right)} .
$$

By Eq. (32), the expansion coefficients $b_{N}(\boldsymbol{m})$ are sums of signs of permutations and therefore integers. The proof of the lemma is concluded by the following observation: if $m_{1} \leq \ldots \leq m_{N}$ is $N$-admissible and irreducible,

$$
\sum_{j=1}^{N}\left(p^{2}(j-1)^{2}-m_{j}^{2}\right) \geq p(N-1) .
$$

Due to $N$-admissibility, we can write $m_{j}=p(j-1)+\nu_{j}-\nu_{j-1}$ with $\nu_{0}=\nu_{N}=0$ and $\nu_{1}, . ., \nu_{N-1} \geq 0$ (see also [FGIL, Property 3 ). $\nu_{k}$ is just $\sum_{j=1}^{k}\left[m_{j}-p(j-1)\right]$. Because of irreducibility, $\nu_{1}, . ., \nu_{k}$ must be strictly positive. In the left-hand side of (46), we insert the expression of $m_{j}$ in terms of $\nu_{k}$ and perform a summation by parts, and obtain Ineq. (46).

Now recall that $q>0$ if and only if $\sum \alpha_{n} r^{n}=1$ and $\sum n \alpha_{n} r^{n}<\infty$. The crucial observation is that these two conditions are automatically fulfilled when the generating series of $\left(\alpha_{n}\right)$ has a radius of convergence $R_{\alpha}$ strictly larger than the radius of convergence $r$ of the power series with coefficients $\left(C_{n}\right)$. Therefore we are going to compare domains of convergence.

For a monomer system with $\alpha_{1}=1$ (and $\alpha_{n}=0$ for $n \geq 2$ ), the quantities are trivial to compute:

$$
C_{n} \equiv 1, \quad r=1, \quad R_{\alpha}=\infty, \quad q=1 .
$$

We will show that on sufficiently thin cylinders, the quantities $r, R_{\alpha}, q$ take values close to the monomer values (47). For this purpose it is useful to keep track of the $\gamma$-dependence in the notation. By Lemma 5 and Eq. (37), the activity and the normalization constants are polynomials of $e^{-\gamma^{2}}$ with coefficients in $\mathbb{N}$. Therefore we write $\alpha_{n}\left(e^{-\gamma^{2}}\right), C_{n}\left(e^{-\gamma^{2}}\right)$. The power series

$$
C\left(t, e^{-\gamma^{2}}\right):=1+\sum_{n=1}^{\infty} C_{n}\left(e^{-\gamma^{2}}\right) t^{n}, \quad A\left(t, e^{-\gamma^{2}}\right):=t+\sum_{n=2}^{\infty} \alpha_{n}\left(e^{-\gamma^{2}}\right) t^{n}
$$

are actually power series of two variables, $t$ and $u=e^{-\gamma^{2}}$. They have nonnegative integer coefficients and are related through

$$
C(t, u)=\frac{1}{1-A(t, u)},
$$

see Eq. (40). The curves $r=r(u)$ and $R_{\alpha}=R_{\alpha}(u)$ delimit the domains of convergence of $C(t, u)$ and $A(t, u)$, see Fig. 3 ,

The following theorem states that the curve $r(u)$ stays strictly below $R(u)$, at least for small $u$ (large $\gamma$ ), and $r(u)$ and $q(u)$ converge to the monomer values $r=q=1$ in the limit of thin cylinders $(u \rightarrow 0)$. 


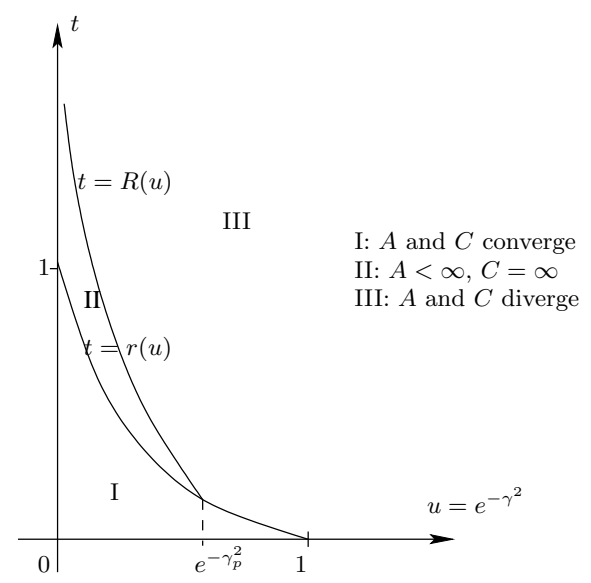

Fig. 3. Domains of convergence of $A(t, u)$ and $C(t, u)$ for $p \geq 2$. The curve $R(u)$ delimits the domain of convergence of $A, r(u)$ the domain of convergence of $C$. Both series diverge when $u \geq 1$. We know that $r(u)=1+O(u)$ and $R(u) \geq$ const $\cdot u^{-p}$ as $u \rightarrow 0$. When $u<\exp \left(-\gamma_{p}^{2}\right)$, $r(u)<R(u)$. It is an open question whether the curves $r$ and $R$ touch for some $u=\exp \left(-\gamma_{p}^{2}\right)$ strictly below 1 .

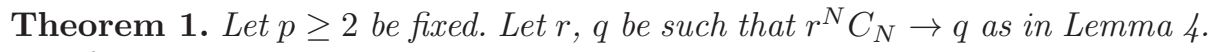
The following holds:

1. There exists a $\gamma_{p}>0$ such that for $\gamma>\gamma_{p}, r<R_{\alpha}$.

2. The functions $] \gamma_{p}, \infty[\ni \gamma \mapsto r, q$ are analytic and strictly positive. As $\gamma \rightarrow \infty$,

$$
r=1+O\left(e^{-\gamma^{2}}\right), \quad q=1+O\left(e^{-\gamma^{2}}\right) .
$$

Proof. 1. Let $0<u \leq v<1$. By Lemma 5 ,

$$
\alpha_{n}(u)=\sum_{m \geq p(n-1)} b_{m n} u^{m} \leq\left(\frac{u}{v}\right)^{p(n-1)} \alpha_{n}(v)
$$

with suitable non-negative integers $b_{m n}$. It follows that $u^{p} R_{\alpha}(u) \geq v^{p} R_{\alpha}(v)$. We fix $v$ and let $u \rightarrow 0$. Since $R_{\alpha}(v) \geq r(v)>0$, we obtain that $R_{\alpha}(u)$ goes to infinity when $u \rightarrow 0$, as expected from Eq. (47). On the other hand, we know that $C_{N} \geq \alpha_{1}^{N}=1$, hence $r(u) \leq 1$. Thus for sufficiently small $u=\exp \left(-\gamma^{2}\right)$, $r(u) \leq 1<R_{\alpha}(u)$.

2. The positivity of $r$ was proved in Lemma 4 The positivity of $q$ is a consequence of $r<R_{\alpha}$. Now, notice that the power series $A(t, u)$ defines a holomorphic function of two complex variables in the domain $|t|<R_{\alpha}(|u|)$. For $0 \leq u<\exp \left(-\gamma_{p}^{2}\right), r(u)$ is the unique solution of

$$
A(r(u), u)=1 .
$$

By Lemma $5(t, 0)=t$. Thus the thin cylinder limit corresponds to the point $(r(0), 0)=(1,0)$. We can apply an implicit function theorem for holomorphic functions to obtain the analyticity of $r$. The analyticity of $q$ follows from

$$
q(u)=\left(\left(\partial_{t} A\right)(r(u), u)\right)^{-1} .
$$


Both $r(u)$ and $q(u)$ can be extended to holomorphic functions in a complex neighborhood of $u=0$ and take the value 1 at 0 , whence Eq. (48).

Theorem 1 is the central technical result of the present work, as all our results on the one particle density will rely on the condition $q>0$.

3.4. Thermodynamic limits of correlation functions and symmetries. In this subsection, we show that Laughlin's state has a unique thermodynamic limit (Theorem 2) and show that the limiting state is periodic in the axial direction, with $p \gamma$ as one of its periods. The proof that $p \gamma$ is actually the smallest period is deferred to the next subsection.

The results presented here hold provided $\lim r^{n} C_{n}=q>0$, i.e., the associated renewal process has finite mean. From the previous subsection, we know that this condition is indeed fulfilled on sufficiently thin cylinders.

In the following, $\mathcal{A}$ is the $C^{*}$-algebra generated by the fermionic creation and annihilation operators $c^{*}(f), c(g)$, with $f, g \in L^{2}(\mathbb{R} \times[0,2 \pi / \gamma])$. The operators associated with the lowest Landau level basis state $\psi_{k}$ are denoted $c_{k}^{*}, c_{k}$.

Theorem 2 (Existence of the thermodynamic limit). Suppose $r^{n} C_{n} \rightarrow$ $q>0$. There is a state $\langle\cdot\rangle$ such that for every sequence of integers $\left(a_{N}\right)$ such that $a_{N} \rightarrow \infty$ and $N+a_{N} \rightarrow \infty$, the states associated with the shifted Laughlin functions $\tilde{\Psi}_{N}=t\left(a_{N} p \gamma \boldsymbol{e}_{\mathrm{x}}\right)^{\otimes N} \Psi_{N}$ converge to $\langle\cdot\rangle$ : for all $a \in \mathcal{A}$,

$$
\langle a\rangle_{N}=\frac{1}{C_{N}}\left\langle\tilde{\Psi}_{N}, a \tilde{\Psi}_{N}\right\rangle \underset{N \rightarrow \infty}{\longrightarrow}\langle a\rangle .
$$

Proof. For $L=\left\{\ell_{1}<. .<\ell_{r}\right\} \subset \mathbb{Z}$, let $c_{L}:=c_{\ell_{1}} . . c_{\ell_{r}}$ and $c_{L}^{*}:=\left(c_{L}\right)^{*}$. It is enough to prove the convergence (50) for operators $a=c_{L^{\prime}}^{*} c_{L}$ with $\left|L^{\prime}\right|=|L|$. The key idea of the proof is to show a formula similar to the one given in Prop. 1 for the solvable model, and then to use the asymptotics of the normalization constants, just as we did in the proof of Corollary 1 Let $b_{N}:=N+a_{N}$. We will see that $\left\langle c_{L^{\prime}}^{*} c_{L}\right\rangle_{N}$ can be written as

$$
\left\langle c_{L^{\prime}}^{*} c_{L}\right\rangle_{N}=\sum_{n=1}^{N} \sum_{j=a_{N}}^{b_{N}-n} \frac{C_{j-a_{N}} C_{b_{N}-j-n}}{C_{N}} f_{n}\left(L^{\prime}-p j, L-p j\right)
$$

for a suitable $N$-independent family of functions $\left(f_{n}\right)_{n \in \mathbb{N}}$. We use the notation

$$
L-p j=\left\{\ell_{1}, . ., \ell_{r}\right\}-p j=\left\{\ell_{1}-p j, . ., \ell_{r}-p j\right\} .
$$

The $f_{n}$ 's have finite support,

$$
f_{n}\left(L^{\prime}, L\right) \neq 0 \Rightarrow L \cup L^{\prime} \subset\{0, . ., p n-p\}
$$

and non-negative "diagonal" values:

$$
\forall L \subset \mathbb{Z}: f_{n}(L, L) \geq 0 .
$$

For fixed $j$ and $n, C_{j-a_{N}} C_{b_{N}-j-n} / C_{N} \rightarrow q r^{n}$ due to $C_{N} r^{N} \rightarrow q>0$. Thus formally, the right-hand side of Eq. (51) converges to

$$
\left\langle c_{L^{\prime}}^{*} c_{L}\right\rangle=\sum_{n=1}^{\infty} q r^{n} \sum_{j=-\infty}^{\infty} f_{n}\left(L^{\prime}-p j, L-p j\right),
$$


provided the series converges.

We prove the theorem in three steps. First, we define the auxiliary functions $f_{n}$ and prove the representation (51) of the correlation functions. Second, we look at "diagonal" correlation functions $\left(L^{\prime}=L\right)$ and show that the series (53) is bounded and equals the limit of correlation functions:

$$
\left\langle c_{L}^{*} c_{L}\right\rangle \leq 1, \quad \lim _{N \rightarrow \infty}\left\langle c_{L}^{*} c_{L}\right\rangle_{N}=\left\langle c_{L}^{*} c_{L}\right\rangle
$$

As a last step, we turn to off-diagonal values $\left(L^{\prime} \neq L\right)$. We prove that the series (53) is absolutely convergent:

$$
\sum_{j, n} q r^{n}\left|f_{n}\left(L^{\prime}-p j, L-p j\right)\right| \leq\left\langle c_{L}^{*} c_{L}\right\rangle^{1 / 2}\left\langle c_{L^{\prime}}^{*} c_{L^{\prime}}\right\rangle^{1 / 2} \leq 1
$$

and show that $\left\langle c_{L^{\prime}}^{*} c_{L}\right\rangle_{N} \rightarrow\left\langle c_{L^{\prime}}^{*} c_{L}\right\rangle$. Note that once we know that Eq. (53) defines a state on $\mathcal{A}$, the inequality (54) with absolute value bars outside the sum is just Cauchy-Schwarz for the state $\langle\cdot\rangle$.

1. Representation of correlation functions. Let $L^{\prime}, L \subset \mathbb{Z}$ with $\left|L^{\prime}\right|=|L|$. We start with the representation

$$
\left\langle\Psi_{N}, c_{L^{\prime}}^{*} c_{L} \Psi_{N}\right\rangle=\sum_{\boldsymbol{m}^{\prime}, \boldsymbol{m}} \overline{a_{N}\left(\boldsymbol{m}^{\prime}\right)} a_{N}(\boldsymbol{m})\left\langle\psi_{m_{1}^{\prime}} \wedge . . \wedge \psi_{m_{N}^{\prime}}, c_{L^{\prime}}^{*} c_{L} \psi_{m_{1}} \wedge . . \wedge \psi_{m_{N}}\right\rangle
$$

The sum ranges over $N$-admissible sequences $\boldsymbol{m}, \boldsymbol{m}^{\prime}$. Suppose $\boldsymbol{m}$ and $\boldsymbol{m}^{\prime}$ have common renewal points $s, t$ such that $L \cup L^{\prime} \subset\{p s, . ., p t-p\}$. Then

$$
\begin{aligned}
\left\langle\psi_{m_{1}^{\prime}} \wedge . \wedge \wedge \psi_{m_{N}^{\prime}}, c_{L^{\prime}}^{*} c_{L} \psi_{m_{1}} \wedge . . \wedge \psi_{m_{N}}\right\rangle & \\
=\prod_{j \in\{1, . ., s\} \cup\{t, \ldots, N\}} \sum_{m_{j}, m_{j}^{\prime}}\left|a_{s}\left(\boldsymbol{m}_{1}^{s}\right)\right|^{2}\left|a_{N-t}\left(\boldsymbol{m}_{t+1}^{N}-p t\right)\right|^{2} & \frac{a_{t-s}\left(\boldsymbol{m}_{s+1}^{\prime t}-p s\right)}{a_{t-s}}\left(\boldsymbol{m}_{s+1}^{t}-p s\right) \\
& \quad\left\langle\psi_{m_{s+1}^{\prime}} \wedge . . \wedge \psi_{m_{t}^{\prime}}, c_{L^{\prime}}^{*} c_{L} \psi_{m_{s+1}} \wedge . . \wedge \psi_{m_{t}}\right\rangle .
\end{aligned}
$$

Let $\mathcal{M}$ be the set of pairs $\left(\boldsymbol{m}, \boldsymbol{m}^{\prime}\right)$ such that

1. $\boldsymbol{m}, \boldsymbol{m}^{\prime}$ are both $N$-admissible;

2. $\boldsymbol{m}$ and $\boldsymbol{m}^{\prime}$ have no common renewal point $s$ below or above $p^{-1}\left(L \cup L^{\prime}\right)$.

By " $s$ is below $p^{-1}\left(L \cup L^{\prime}\right)$ " we mean $L \cup L^{\prime} \subset\{0, . ., p s-p\}$, and we say" $s$ is above $p^{-1}\left(L \cup L^{\prime}\right)$ " when $L \cup L^{\prime} \subset\{p s, . ., p n-p\}$. The set $\mathcal{M}$ consists of the pairs $\left(\boldsymbol{m}, \boldsymbol{m}^{\prime}\right)$ for which no simplification of the type (56) is possible.

$f_{N}\left(L^{\prime}, L\right)$ is defined by the sum (55), except that the summation includes only $\left(\boldsymbol{m}, \boldsymbol{m}^{\prime}\right)$ from $\mathcal{M}$. With this definition, combining (55) and (56) we obtain (51).

2. "Diagonal" correlation functions. For $L=L^{\prime}$, the definition of $f_{n}$ gives

$$
f_{N}(L, L)=\sum_{\boldsymbol{m}}^{\prime}\left|a_{N}(\boldsymbol{m})\right|^{2} \chi_{L \subset\left\{m_{1}, . ., m_{N}\right\}}
$$


where the sum ranges over $N$-admissible sequences that are $L$-irreducible. In particular, $f_{N}(L, L) \geq 0$. Moreover, if $f_{N}(L, L) \neq 0$, there exists an $N$-admissible sequence $\boldsymbol{m}$ such that $L \subset\left\{m_{1}, . ., m_{N}\right\}$, whence $L \subset\{0, . ., p N-p\}$.

Let $d \in \mathbb{N}$. From (51) and $f_{n}(L, L) \geq 0$ we get

$$
\sum_{n=1}^{d} \sum_{j=a_{N}}^{b_{N}-j-n} \frac{C_{j-a_{N}} C_{b_{N}-j-n}}{C_{N}} f_{n}(L-p j, L-p j) \leq\left\langle c_{L}^{*} c_{L}\right\rangle_{N} \leq 1
$$

If $f_{n}(L-p j, L-p j) \neq 0$ we must have $L-p j \subset\{0, . ., p n-p\}$, thus only a finite, $N$-independent number of $j$ 's contribute to the sum and we can take the limit $N \rightarrow \infty$, which gives

$$
\sum_{n=1}^{d} \sum_{j=-\infty}^{\infty} q r^{n} f_{n}(L-p j, L-p j) \leq 1 .
$$

Letting $d \rightarrow \infty$, we obtain the bound $\left\langle c_{L}^{*} c_{L}\right\rangle \leq 1$. The proof of $\left\langle c_{L}^{*} c_{L}\right\rangle_{N} \rightarrow\left\langle c_{L}^{*} c_{L}\right\rangle$ is then completed by an $\epsilon / 3$ argument. We leave the details to the reader and mention only a useful inequality on quotients of normalization constants. Using the supermultiplicativity of $\left(C_{N}\right), 0<C_{n} r^{n} \leq 1$ and $C_{n} r^{n} \rightarrow q>0$, we get $\inf _{n} r^{n} C_{n}=: c>0$ and

$$
\frac{C_{j} C_{N-j-n}}{C_{N}} \leq \frac{C_{N-n}}{C_{N}} \leq \frac{r^{-(N-n)}}{c r^{-N}}=\frac{1}{c} r^{n} .
$$

3. "Off-diagonal" correlation functions $\left(L \neq L^{\prime}\right)$. The procedure is similar to step 2. but the analogue of the bound (57) is slightly more delicate to obtain. Let $d \in \mathbb{N}$. Then

$$
\begin{aligned}
\sum_{n=1}^{d} \sum_{j=a_{N}}^{b_{N}-n} & \frac{C_{j-a_{N}} C_{b_{N}-n-j}}{C_{N}}\left|f_{n}\left(L^{\prime}-p j, L-p j\right)\right| \\
& \leq \sum_{n=1}^{N} \sum_{j=a_{N}}^{b_{N}-n} \frac{C_{j-a_{N}} C_{b_{N}-n-j}}{C_{N}}\left|f_{n}\left(L^{\prime}-p j, L-p j\right)\right| \\
& =C_{N}^{-1} \sum_{\boldsymbol{m}_{\boldsymbol{m}^{\prime}}}\left|a_{N}\left(\boldsymbol{m}^{\prime}\right) a_{N}(\boldsymbol{m})\left\langle\psi_{m_{1}^{\prime}} \wedge . \wedge \wedge \psi_{m_{N}}, c_{L^{\prime}}^{*} c_{L} \psi_{m_{1}} \wedge . \wedge \psi_{m_{N}}\right\rangle\right| \\
& =C_{N}^{-1} \sum_{K \subset \mathbb{Z},|K|=N-|L|}\left|a_{N}\left(L^{\prime} \cup K\right) a_{N}(L \cup K)\right| \\
& \leq C_{N}^{-1}\left(\sum_{K}\left|a_{N}\left(L^{\prime} \cup K\right)\right|^{2}\right)^{1 / 2}\left(\sum_{K}\left|a_{N}(L \cup K)\right|^{2}\right)^{1 / 2} \\
& =\left\langle c_{L^{\prime}}^{*} c_{L^{\prime}}\right\rangle_{N}^{1 / 2}\left\langle c_{L}^{*} c_{L}\right\rangle_{N}^{1 / 2} \leq 1 .
\end{aligned}
$$

The notation $a_{N}(L \cup K)$ refers to the amplitude of the increasing sequence obtained by rearranging the elements of $L \cup K$. Letting first $N$ and then $d$ go to infinity, we obtain the bound (54). The convergence $\left\langle c_{L^{\prime}}^{*} c_{L}\right\rangle_{N} \rightarrow\left\langle c_{L^{\prime}}^{*} c_{L}\right\rangle$ can be shown with an $\epsilon / 3$ argument. 
Remark. The representation (53) of correlation functions does not lend itself to a simple interpretation. However, it leads to a very nice formula for the one particle density. Let $\hat{n}_{k}:=c_{k}^{*} c_{k}$ be the number operator for the lowest Landau level state $\psi_{k}$. The quantity $f_{n}(\{k\},\{k\})$ equals $\left\langle u_{X_{0}}, \hat{n}_{k} u_{X_{0}}\right\rangle$ with the polymer $X_{0}=\{0, . ., p n-p\}$. Therefore Eq. (53) may be rewritten as

$$
\left\langle\hat{n}_{k}\right\rangle=\sum_{X} \rho^{\mathrm{P}}(X) v_{X}(k)
$$

with $\rho^{\mathrm{P}}(X)=q \alpha_{N(X)} r^{N(X)}$ and $v_{X}(k)=\left\langle u_{X}, \hat{n}_{k} u_{X}\right\rangle /\left\|u_{X}\right\|^{2}$. The sum is over all polymers $X=\{j, . ., j+n-1\}, j \in \mathbb{Z}, n \in \mathbb{N}$. This formula has an intuitive probabilistic interpretation: $v_{X}(k)$ is the probability of finding a particle in the "site" $k$, given that $k$ is contained in the polymer $X$ (or, strictly speaking, in $\{p \min X, \ldots, p \max X\})$, which happens with probability $\rho^{\mathrm{P}}(X)^{5}$.

Together with a similar formula for two-point correlations $\left\langle\hat{n}_{k} \hat{n}_{j}\right\rangle$, Eq. (58) will serve as a useful guide in Sect. 3.5 when we investigate clustering properties.

Now let us turn to the symmetries of Laughlin's state. Let $\tau_{x}$ be the automorphism of the algebra $\mathcal{A}$ associated with the magnetic translation $t\left(\gamma \mathbf{e}_{x}\right)$. Let $\tau_{y}^{a}$ be the morphism associated with the translation $t\left(a \mathbf{e}_{y}\right)$ in the $y$-direction, and $\tau_{s}$ the morphism induced by the reversal $\left(s_{0} \psi\right)(z)=\psi(-z)$.

Proposition 3 (Symmetries). The state $\omega(\cdot)=\langle\cdot\rangle$ of the previous theorem is invariant with respect to reversal, translations in the $x$-direction by multiples of $p \gamma$, and arbitrary translations in the $y$-direction:

$$
\forall n \in \mathbb{Z}, \forall a \in \mathbb{R}: \omega=\omega \circ \tau_{s}=\omega \circ \tau_{x}^{n p}=\omega \circ \tau_{y}^{a} .
$$

Proof. The invariance with respect to $y$-translations is a direct consequence of the fact that $\Psi_{N}$ has a definite $y$-momentum, see Eq. (21). The reversal invariance follows from the invariance for finitely many particles (22), see also ŠWK. The periodicity with respect to magnetic translations in the direction along the cylinder follows from the representation (53) of correlation functions.

Theorem 2 and Prop. 3 lead to a simple corollary on the one-particle density:

Corollary 2. Let $\rho_{N}(z)$ be the one-particle density of Laughlin's state $\Psi_{N}$. Under the assumptions of Theorem 2 , the shifted density converges pointwise to the one-particle density $\rho(z)$ of the limiting state $\langle\cdot\rangle$ :

$$
\lim _{N \rightarrow \infty} \rho_{N}(z-p\lfloor N / 2\rfloor \gamma)=\rho(z), \quad \rho(z)=\sum_{k=-\infty}^{\infty}\left\langle\hat{n}_{k}\right\rangle\left|\psi_{k}(z)\right|^{2} .
$$

The density is independent of the coordinate $y=\operatorname{Im} z$ around the cylinder. The density as well as the occupation numbers are periodic and reversal invariant:

$$
\begin{array}{rlrl}
\rho(x+p \gamma) & =\rho(x), \quad\left\langle\hat{n}_{k+p}\right\rangle & =\left\langle\hat{n}_{k}\right\rangle, \\
\rho(-x) & =\rho(x), \quad\left\langle\hat{n}_{-k}\right\rangle=\left\langle\hat{n}_{k}\right\rangle .
\end{array}
$$

\footnotetext{
5 The notation $\rho^{\mathrm{P}}$ refers to polymer correlation functions as defined in GK. Later, we shall use not only $\rho^{\mathrm{P}}(X)$ but also $\rho^{\mathrm{P}}(X, Y)$, see p. 28
} 
Note that weak*-convergence of the state $\langle\cdot\rangle_{N}$ is replaced with pointwise convergence of the one-particle density. This uses the representation of the density as a sum of Gaussians with occupation numbers as coefficients as in Eq. (23). Due to the good localization of the Gaussians, summation and limits can be interchanged, whence Eq. (59).

3.5. Symmetry breaking and clustering. This subsection contains the second part of the main results of this paper: Theorem 3 shows that on sufficiently thin cylinders, $p \gamma$ is actually the smallest period of the limiting state $\langle\cdot\rangle$ as well as the one-particle density $\rho$ of the previous subsection. Thus the state $\langle\cdot\rangle$ has a larger minimal period than the Hamiltonian describing interacting electrons in a magnetic field, whose ground states it is supposed to approximate. In this sense, there is symmetry breaking.

In addition, we prove that the state $\langle\cdot\rangle$ is mixing with respect to magnetic translations in the direction of the cylinder axis (Theorem 4).

Theorem 3 (Symmetry breaking). Suppose $C_{n} r^{n} \rightarrow q>0$. Let $\rho(x)$ be the infinite cylinder density from Cor. 囵. Then on sufficiently thin cylinders, $p \gamma$ is the smallest period of $\rho(x)$.

Proof. Due to Eq. (59) and Lemma 2, it is enough to look at the occupation numbers. We will show that

$$
\left\langle\hat{n}_{k}\right\rangle= \begin{cases}1+O\left(\exp \left(-\gamma^{2}\right)\right), & \text { if } k \in p \mathbb{Z} \\ O\left(\exp \left(-\gamma^{2}\right)\right), & \text { else. }\end{cases}
$$

Thus for sufficiently large $\gamma$, the sequence of occupation numbers has $p$ as the smallest period and $p \gamma$ is the smallest period of the one-particle density.

The idea behind (60) is that the thin cylinder limit is at the same time a monomer limit, see Lemma 5 and p. 22. The wave function corresponding to a pure monomer system, for $N$ particles, is

$$
u_{\{0\}} \wedge u_{\{1\}} \wedge . . \wedge u_{\{N-1\}}=\psi_{0} \wedge \psi_{p} \wedge . . \wedge \psi_{p N-p} .
$$

In the limit $N \rightarrow \infty$, the corresponding monomer occupation numbers $\left\langle\hat{n}_{k}\right\rangle_{\text {mon }}$ equal 1 if $k$ is a multiple of $p$, and 0 otherwise.

Eq. (60) now is a consequence of the following observation: the occupation numbers $\left\langle\hat{n}_{k}\right\rangle$ are functions of $v=\exp \left(-\gamma^{2}\right)$ that can be extended to holomorphic functions of $v$ in a complex neighborhood of the monomer point $v=0$. This can be shown with the representation

$$
\left\langle\hat{n}_{k}\right\rangle=\sum_{n=1}^{\infty} q r^{n} \sum_{j=-\infty}^{\infty} f_{n}(\{k-p j\},\{k-p j\})=\sum_{n=1}^{\infty} q r^{n} g_{n}(k),
$$

see Eq. (53). $g_{n}(k)$ is a polynomial of $\exp \left(-\gamma^{2}\right)$, and $q$ and $r$ are analytic functions of $\exp \left(-\gamma^{2}\right)$. We can adapt the procedure used in the proof of Theorem 1 and deduce the analyticity of $\left\langle\hat{n}_{k}\right\rangle$ for small $u$. 
Remarks. 1. The monomer state (61) is the Tao-Thouless state, corresponding to the reference configuration $\boldsymbol{m}^{\mathrm{TT}}$ on $\mathrm{p}$. 15. The fact that Laughlin's wave function for a fixed, finite number of particles on very thin cylinders approaches the TaoThouless state has been observed by Rezayi and Haldane $\mathrm{RH}$. The novelty here is twofold: first, the limits $N \rightarrow \infty$ and $\gamma \rightarrow \infty$ can be interchanged; second, the periodicity survives for small but non-vanishing cylinder radius.

2. If we assimilate orbitals $\psi_{k}$ with lattice sites $k \in \mathbb{Z}$, the restriction of the state $\omega$ to the algebra generated by the number operators $\hat{n}_{k}$ can be described by a probability distribution $P$ on particle configurations on $\mathbb{Z}$. Adapting techniques from $\mathrm{AM}, \mathrm{AGL}$, one can show that the probability measures corresponding to $\omega$ and the shifted states $\omega \circ \tau_{x}, \ldots, \omega \circ \tau_{x}^{p-1}$ are mutually singular. This result holds provided $r^{n} C_{n} \rightarrow q>0$ and the second moment $\sum_{n} n^{2} \alpha_{n} r^{n}$ is finite. Again, this condition is fulfilled when $\gamma$ is large enough. As a consequence, the $p$ quantummechanical states $\omega, . ., \omega \circ \tau_{x}^{p-1}$ are not only distinct, but also orthogonal in the sense of $[\mathrm{BR}]$, Def. 4.1.20.

Now we come to clustering properties. Before we state our result in its general form, let us have a look at two-point correlations $\left\langle\hat{n}_{k} \hat{n}_{l}\right\rangle$, where $\hat{n}_{k}=c_{k}^{*} c_{k}$ is the number operator for the state $\psi_{k}$. In the spirit of the remark on p. 26. $\left\langle\hat{n}_{k} \hat{n}_{l}\right\rangle$ may be interpreted as the probability of finding a particle in the site $k$ and another particle in the site $l$. In fact, we have a formula analogous to Eq. (58) for the one-particle density. Define $\rho^{\mathrm{P}}(X)$ and $v_{X}(k)$ as on p. 26. Let $v_{X}(k, l):=\left\langle u_{X}, \hat{n}_{k} \hat{n}_{l} u_{X}\right\rangle /\left\|u_{X}\right\|^{2}$ and

$$
\rho^{\mathrm{P}}(X, Y)=q r^{N(X)} \alpha_{N(X)} r^{d(X, Y)} C_{d(X, Y)} r^{N(Y)} \alpha_{N(Y)}
$$

where $X$ is to the left hand side of $Y$, separated from $Y$ by the distance

$$
d(X, Y):=\min Y-\max X-1 \geq 0
$$

$v_{X}(k, l)$ is the probability of finding particles in the sites $k$ and $l$ given that $k$ and $l$ are contained in $[p \min X, p \max X]$, while $\rho^{\mathrm{P}}(X, Y)$ is the probability of finding the rods $X$ and $Y$.

Suppose that $k<l$. Then the diagonal two-point correlation equals

$$
\left\langle\hat{n}_{k} \hat{n}_{l}\right\rangle=\sum_{X<Y} \rho^{\mathrm{P}}(X, Y) v_{X}(k) v_{Y}(l)+\sum_{X} \rho^{\mathrm{P}}(X) v_{X}(k . l) .
$$

Again, this formula has an intuitive probabilistic interpretation. The two sums correspond to the two different situations that $k$ and $l$ are contained in two distinct polymers (first sum) or in the same polymer (second sum).

When $k$ and $l$ are far apart, the probability that they are in the same polymer is small. On the other hand, when $X$ and $Y$ are far apart, we may write

$$
\rho^{\mathrm{P}}(X, Y) \simeq q r^{N(X)} \alpha_{N(X)} q r^{N(Y)} \alpha_{N(Y)}=\rho^{\mathrm{P}}(X) \rho^{\mathrm{P}}(Y) .
$$

Therefore we expect that $\left\langle\hat{n}_{k} \hat{n}_{l}\right\rangle$ is approximately the same as $\left\langle\hat{n}_{k}\right\rangle\left\langle\hat{n}_{l}\right\rangle$ when $k$ and $l$ are far apart: Laughlin's state inherits clustering properties from the polymer correlations $\rho^{\mathrm{P}}$. 
Theorem 4 (Clustering). Suppose $r^{n} C_{n} \rightarrow q>0$. Then the state $\langle\cdot\rangle$ of Theorem 0 is mixing with respect to the shifts $\tau_{x}^{n p}, n \in \mathbb{Z}$ :

$$
\forall a, b \in \mathcal{A}: \lim _{n \rightarrow \infty}\left\langle a \tau_{x}^{p n}(b)\right\rangle=\langle a\rangle\langle b\rangle .
$$

Proof. We use the notation from the proof of Theorem 2, It is enough to check (63) for operators $a=c_{L^{\prime}}^{*} c_{L}, b=c_{K^{\prime}}^{*} c_{K}$ with $L, L^{\prime}, K, K^{\prime} \subset \mathbb{Z}$. Because of particle number conservation and $y$-invariance, the only interesting case is

$$
|L|=\left|L^{\prime}\right|, \quad|K|=\left|K^{\prime}\right|, \quad \sum_{k \in K^{\prime} \cup L^{\prime}} k=\sum_{k \in K \cup L} k .
$$

In the following we will assume that (64) holds and show that $\langle a b\rangle-\langle a\rangle\langle b\rangle$ is small when $L \cup L^{\prime}$ is far to the left of $K \cup K^{\prime}$. The main idea is to generalize the formula (62) for two-point correlations. We will see that

$$
\begin{aligned}
\left\langle c_{L^{\prime}}^{*} c_{L} c_{K^{\prime}}^{*} c_{K}\right\rangle & =F+G \\
F & =\sum_{X<Y} q r^{N(X)+N(Y)} f_{X}\left(L^{\prime}, L\right) C_{d(X, Y)} r^{d(X, Y)} f_{Y}\left(K^{\prime}, K\right) \\
G & =\sum_{X} q r^{N(X)} g_{X}\left(L^{\prime}, L ; K^{\prime}, K\right)
\end{aligned}
$$

where $d(X, Y)=\min Y-\max X-1$ and the functions $f_{X}, g_{X}$ will be defined later. Similarly,

$$
\left\langle c_{L^{\prime}}^{*} c_{L}\right\rangle\left\langle c_{K^{\prime}}^{*} c_{K}\right\rangle=\sum_{X, Y} q^{2} r^{N(X)+N(Y)} f_{X}\left(L^{\prime}, L\right) f_{Y}\left(K^{\prime}, K\right) .
$$

The theorem is proved by making the following arguments precise: Suppose $L \cup L^{\prime}$ and $K \cup K^{\prime}$ are far away. Then, intuitively, the main contributions to $F$ in (65) come from polymers $X, Y$ separated by a large distance $d(X, Y)$. Since $r^{n} C_{n} \rightarrow q$, we expect that $F$ is close to the righthand side of Eq. (66). The second contribution, $G$, in Eq. (65) will be bounded by the probability for finding a long polymer, which is small. Thus

$$
\left\langle c_{L^{\prime}}^{*} c_{L} c_{K^{\prime}}^{*} c_{K}\right\rangle \approx F \approx\left\langle c_{L^{\prime}}^{*} c_{L}\right\rangle\left\langle c_{K^{\prime}}^{*} c_{K}\right\rangle .
$$

Now we define $f_{X}$ and $g_{X}$, and prove Eqs. (65) and (66). The argument resembles step 1 . in the proof of Theorem 2 therefore we shall only give the key elements. Let $\mathcal{M}^{\prime}$ be the set of pairs $\left(\boldsymbol{m}, \boldsymbol{m}^{\prime}\right)$ such that:

1. $\boldsymbol{m}$ and $\boldsymbol{m}^{\prime}$ are $N$-admissible.

2. $\boldsymbol{m}$ and $\boldsymbol{m}^{\prime}$ have no common renewal point $s$

(a) below or above $p^{-1}\left(L \cup L^{\prime} \cup K \cup K^{\prime}\right)$,

(b) between $p^{-1}\left(L \cup L^{\prime}\right)$ and $p^{-1}\left(K \cup K^{\prime}\right)$.

We say that " $s$ is between $p^{-1}\left(L \cup L^{\prime}\right)$ and $p^{-1}\left(K \cup K^{\prime}\right)$ " if $L \cup L^{\prime}$ is contained in $\{0, . ., p s-p\}$ and $K \cup K^{\prime}$ in $\{p s, . ., p N-p\}$. The set $\mathcal{M}^{\prime}$ is a subset of the set $\mathcal{M}$ introduced on p. 24 for the definition of $f_{N}$. Let

$$
\begin{aligned}
& g_{N}\left(L^{\prime}, L ; K^{\prime}, K\right) \\
& :=\sum_{\left(\boldsymbol{m}, \boldsymbol{m}^{\prime}\right) \in \mathcal{M}^{\prime}} \overline{a_{N}\left(\boldsymbol{m}^{\prime}\right)} a_{N}(\boldsymbol{m})\left\langle\psi_{m_{1}^{\prime}} \wedge . . \wedge \psi_{m_{N}^{\prime}}, c_{L^{\prime}}^{*} c_{L} c_{K^{\prime}}^{*} c_{K} \psi_{m_{1}} \wedge . . \wedge \psi_{m_{N}}\right\rangle .
\end{aligned}
$$




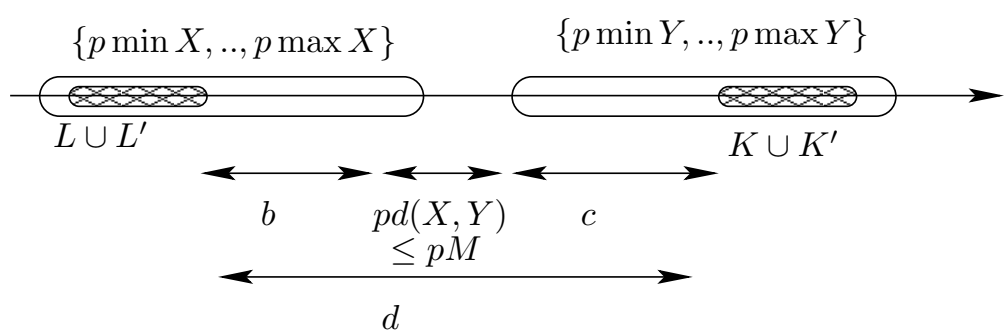

Fig. 4. Visualization of Ineqs. (69) and (70). If $L \cup L^{\prime}$ is contained in $[p \min X, p \max X]$ and $K \cup K^{\prime}$ is contained in $[p \min Y, p \max Y]$, and if the distance $d$ between $L \cup L^{\prime}$ and $K \cup K^{\prime}$ is large but the distance $d(X, Y)$ between $X$ and $Y$ is small, $b$ or $c$ must be large.

The product rule from Lemma 3 gives

$$
\begin{aligned}
f_{N} & \left(L^{\prime} \cup K^{\prime}, L \cup K\right) \\
& =\sum_{0<i \leq j<N} f_{i}\left(L^{\prime}, L\right) C_{j-i} f_{N-j}\left(K^{\prime}-p j, K-p j\right)+g_{N}\left(L^{\prime}, L ; K^{\prime}, K\right) .
\end{aligned}
$$

For $X=\{j, . ., j+N-1\}$, it is convenient to define

$$
\begin{aligned}
f_{X}\left(L^{\prime}, L\right) & =f_{N}\left(L^{\prime}-p j, L-p j\right), \\
g_{X}\left(L^{\prime}, L ; K^{\prime}, K\right) & =g_{N}\left(L^{\prime}-p j, L-p j ; K^{\prime}-p j, K-p j\right) .
\end{aligned}
$$

Eqs. (65) and (66) hold with these definitions of $f_{X}, g_{X}$, as can be seen with Eqs. (53) and (67).

Next, we estimate $F-\left\langle c_{L^{\prime}}^{*} c_{L}\right\rangle\left\langle c_{K^{\prime}}^{*} c_{K}\right\rangle$. In view of Eqs. (65) and (66), this difference is a sum over polymers $X, Y$ :

$$
\sum_{X, Y} q r^{N(X)+N(Y)} f_{X}\left(L^{\prime}, L\right)\left(C_{d(X, Y)} r^{d(X, Y)}-q\right) f_{Y}\left(K^{\prime}, K\right)
$$

with the convention $C_{n}=0$ for $n<0$. We split the sum in two parts. First, suppose that $X$ is far to the left of $Y$, i.e., $d(X, Y) \geq M$ for some fixed $M \in \mathbb{N}$. The sum over such $(X, Y)$ is bounded by

$$
\begin{aligned}
\sup _{n \geq M}\left|1-q^{-1} r^{n} C_{n}\right|\left(\sum_{X} q r^{N(X)}\left|f_{X}\left(L^{\prime}, L\right)\right|\right) & \left(\sum_{Y} q r^{N(Y)}\left|f_{Y}\left(K^{\prime}, K\right)\right|\right) \\
& \leq \sup _{n \geq M}\left|1-q^{-1} r^{n} C_{n}\right| \underset{M \rightarrow \infty}{\longrightarrow} 0,
\end{aligned}
$$

where we have used Ineq. (54). Second, suppose that $X, Y \subset \mathbb{Z}$ satisfy $d(X, Y) \leq$ $M$ and $f_{X}\left(L^{\prime}, L\right) f_{Y}\left(K^{\prime}, K\right) \neq 0$. Then $X$ or $Y$ must be a long polymer, see Fig. 4. More precisely, (52) can be used to show that

$$
p(\max X+1)-\max L \cup L^{\prime} \geq(d-p M) / 2 .
$$

or

$$
\min \left(K \cup K^{\prime}\right)-p \min Y \geq(d-p M) / 2
$$




\begin{tabular}{lll}
$L^{\prime}=\{1\}$ & $K^{\prime}=\{8\}$ & $\begin{array}{l}\boldsymbol{m}^{\prime}=(1,3,6,8) \\
\text { no renewal point } \\
X_{1}^{\prime}=\{0,1,2,3\}\end{array}$ \\
\hline $\begin{array}{l}\boldsymbol{m}=(0,3,6,9) \\
\text { renewal points } 1,2,3 \\
X_{1}=\{0\}, X_{2}=\{1\}, \\
X_{3}=\{2\}, X_{4}=\{3\}\end{array}$
\end{tabular}

Fig. 5. Evaluation of $G$ in order to estimate $\left\langle c_{1}^{*} c_{8}^{*} c_{0} c_{9}\right\rangle(p=3)$ : the sequences $\boldsymbol{m}^{\prime}, \boldsymbol{m}$ give a non-vanishing contribution to $g_{4}(\{1\},\{8\} ;\{0\},\{9\})$. The vector $\boldsymbol{m}^{\prime}$ has larger partial sums and no renewal points. Each polymer $X$ contributes $N(X)$ particles (filled circles) localized in the discrete volume $\{3 \min X, . ., 3 \max X+2\}$ (boxes).

must hold. The sum (68) over pairs $X, Y$ such that $X$ satisfies Ineq. (69) is bounded by

$$
\left|\sum_{X}^{\prime} q r^{N(X)} f_{X}\left(L^{\prime}, L\right)\right|\left(1+q^{-1}\right)\left|\left\langle c_{K^{\prime}}^{*} c_{K}\right\rangle\right| \leq\left(1+q^{-1}\right)\left|\sum_{X}^{\prime} q r^{N(X)} f_{X}\left(L^{\prime}, L\right)\right|,
$$

where the prime at the sum refers to the constraint (69). By a procedure similar to step 3. of the proof of Theorem 2, one can show that

$$
\left|\sum_{X}^{\prime} q r^{N(X)} f_{X}\left(L^{\prime}, L\right)\right| \leq q \sum_{n \geq(d-p M) / 2 p} n r^{n} \alpha_{n}
$$

The right-hand side represents the probability that a given point is in a polymer of length greater or equal to $(d-p M) / 2 p$. The sum (68) over pairs $(X, Y)$ such that $Y$ satisfies Ineq. (70) can be treated in a similar way. In the end, we obtain

$$
|F-\langle a\rangle\langle b\rangle| \leq \sup _{n \geq M}\left|1-q^{-1} r^{n} C_{n}\right|+2(1+q) \sum_{n \geq(d-p M) / 2 p} n r^{n} \alpha_{n} .
$$

Choosing $M$ of the order of $d / 2 p$, we see that $|F-\langle a\rangle\langle b\rangle|$ goes to 0 as $d$ goes to infinity.

It remains to give a bound on $G$. The idea is to have a closer look at the definition of $g_{n}$ and to show that if $\boldsymbol{m}, \boldsymbol{m}^{\prime}$ give a non-vanishing contribution to the sum, at least one of the vectors has no renewal point between $p^{-1}\left(L \cup L^{\prime}\right)$ and $p^{-1}\left(K \cup K^{\prime}\right)$, see Fig. 5 This is stronger than the original requirement that $\boldsymbol{m}$ and $\boldsymbol{m}^{\prime}$ have no common renewal point between $p^{-1}\left(L \cup L^{\prime}\right)$ and $p^{-1}\left(K \cup K^{\prime}\right)$. As a consequence, one can bound $G$ by the square root of the probability that the space between $p^{-1}\left(K \cup K^{\prime}\right)$ and $p^{-1}\left(L \cup L^{\prime}\right)$ is covered by one single long polymer. The square root comes from a Cauchy-Schwarz inequality and accounts for the fact that one of the sequences $\boldsymbol{m}, \boldsymbol{m}^{\prime}$ may have a renewal point.

We start by looking at the non-vanishing contributions to $g_{n}\left(L^{\prime}, L ; K^{\prime}, K\right)$. Without loss of generality, we may assume $\sum_{k \in L^{\prime}} k \geq \sum_{k \in L} k$ (otherwise, use $\left\langle a^{*}\right\rangle=\overline{\langle a\rangle}$ to interchange $L^{\prime}$ and $\left.L\right)$. Suppose that $\boldsymbol{m}^{\prime}, \boldsymbol{m}$ are $N$-admissible and

$$
\left\langle c_{L^{\prime}} c_{K^{\prime}} \psi_{m_{1}^{\prime}} \wedge . . \wedge \psi_{m_{N}^{\prime}}, c_{L} c_{K} \psi_{m_{1}} \wedge . . \wedge \psi_{m_{N}}\right\rangle \neq 0
$$

We claim that if $s$ is a renewal point of $\boldsymbol{m}^{\prime}$ such that

$$
L \cup L^{\prime} \subset\{0, . ., p s-p\}, \quad K \cup K^{\prime} \subset\{p s, . ., p N-p\},
$$


$s$ is also a renewal point of $\boldsymbol{m}$. To see this, let $M=\left\{m_{1}, . ., m_{N}\right\}$, similarly for $M^{\prime}$. Then we must have

$$
M^{\prime} \backslash\left(K^{\prime} \cup L^{\prime}\right)=M \backslash(K \cup L) .
$$

Intersecting with $\{0, . ., p s-p\}$, we obtain

$$
\left(M^{\prime} \backslash L^{\prime}\right) \cap\{0, . ., p s-p\}=(M \backslash L) \cap\{0, . ., p s-p\} .
$$

In particular,

$$
\sum_{j=1}^{s}\left(m_{j}^{\prime}-m_{j}\right)=\sum_{k \in L^{\prime}} k-\sum_{k \in L} k \geq 0
$$

Thus

$$
0=\sum_{j=1}^{s}\left(m_{j}^{\prime}-p(j-1)\right) \geq \sum_{j=1}^{s}\left(m_{j}-p(j-1)\right) \geq 0 .
$$

The inequality on the right-hand side must be an equality, showing that $s$ is a renewal point of $\boldsymbol{m}$.

Now suppose $\boldsymbol{m}^{\prime}, \boldsymbol{m}$ give a non-vanishing contribution to the sum defining $g_{n}$. They are by definition not allowed to have a common renewal point between $p^{-1}\left(L \cup L^{\prime}\right)$ and $p^{-1}\left(K \cup K^{\prime}\right)$. As we have just shown, this means that $\boldsymbol{m}^{\prime}$ cannot have a renewal point between $p^{-1}\left(L \cup L^{\prime}\right)$ and $p^{-1}\left(K \cup K^{\prime}\right)$. Thus if $X_{1}^{\prime}, . ., X_{D}^{\prime}$ is the partition of $\{0, . ., N-1\}$ determined by the renewal points of $\boldsymbol{m}^{\prime}$, there is a rod $X_{j}^{\prime}$ such that $\left[p \min X_{j}^{\prime}, p \max X_{j}^{\prime}\right]$ intersects both $L^{\prime} \cup L$ and $K^{\prime} \cup K$. It follows that

$$
N\left(X_{j}^{\prime}\right) \geq \min \left(K^{\prime} \cup K\right)-\max \left(L \cup L^{\prime}\right)=d / p .
$$

In the spirit of step 3. in the proof of Theorem 2, we have

$$
\begin{aligned}
& \left|\sum_{n=1}^{N} \sum_{j=0}^{N-n} \frac{C_{j} C_{N-n-j}}{C_{N}} g_{n}\left(L^{\prime}-p j, L-p j ; K^{\prime}-p j, K-p j\right)\right| \\
& \leq C_{N}^{-1} \sum_{m, \boldsymbol{m}^{\prime}}^{\prime}\left|\overline{a_{N}(\boldsymbol{m})} a_{N}\left(\boldsymbol{m}^{\prime}\right)\left\langle\psi_{m_{1}^{\prime}} \wedge . . \wedge \psi_{m_{N}^{\prime}}, c_{L^{\prime}}^{*} c_{L} c_{K^{\prime}}^{*} c_{K} \psi_{m_{1}} \wedge . . \wedge \psi_{m_{N}}\right\rangle\right| \\
& \leq C_{N}^{-1}\left\langle c_{K}^{*} c_{K} c_{L}^{*} c_{L}\right\rangle_{\Psi_{N}}^{1 / 2} \\
& \quad \cdot\left(\sum_{\boldsymbol{m}^{\prime}}^{\prime}\left|a_{N}\left(\boldsymbol{m}^{\prime}\right)\right|^{2}\left\langle\psi_{m_{1}^{\prime}} \wedge . . \wedge \psi_{m_{N}^{\prime}}, c_{L^{\prime}}^{*} c_{L^{\prime}} c_{K^{\prime}}^{*} c_{K^{\prime}} \psi_{m_{1}^{\prime}} \wedge . . \wedge \psi_{m_{N}^{\prime}}\right\rangle\right)^{1 / 2},
\end{aligned}
$$

where $\sum^{\prime}$ is the sum over sequences $\boldsymbol{m}^{\prime}$ having no renewal point between $p^{-1}(L \cup$ $\left.L^{\prime}\right)$ and $p^{-1}\left(K^{\prime} \cup K\right)$. Taking the limit $N \rightarrow \infty$ after shifting the origin to the middle of the cylinder, we get

$$
|G| \leq\left(q \sum_{n \geq d / p} n r^{n} \alpha_{n}\right)^{1 / 2} \underset{d \rightarrow \infty}{\longrightarrow} 0 .
$$

Remark: Rate of convergence. On sufficiently thin cylinders, we know from Theorem 1 that $\sum_{n} \alpha_{n} t^{n}$ has a radius of convergence $R_{\alpha}$ strictly larger than $r$. In this case $r^{n} C_{n}=q+O\left(\left(r / R_{\alpha}\right)^{n}\right)$ and Ineqs. (71) and (72) show that there is actually exponential clustering. 
Acknowledgements. E.H.L. thanks the Alexander von Humboldt Foundation for a research award, based at the Technical University, Berlin. He also thanks the US National Science Foundation for partial support, grant PHY-0652854. S.J. and R.S. thank the DFG for support under grant no. SE 456/7-1.

\section{References}

[AANS] E. Akkermans, J.E. Avron, R. Narevich, and R. Seiler, Boundary Conditions for Bulk and Edge States in Quantum Hall Systems, Eur. Phys. J. B 1 (1998), 117-121.

[AGL] M. Aizenman, S. Goldstein, and J.L. Lebowitz, Bounded Fluctuations and Translation Symmetry Breaking in One-Dimensional Particle Systems, J. Stat. Phys. 103 (2001), 601-618.

[AM] M. Aizenman and P.A. Martin, Structure of Gibbs States of one Dimensional Coulomb Systems, Commun. Math. Phys. 78 (1980), 99-116.

[BK] E.J. Bergholtz and A. Karlhede, One-dimensional theory of the Quantum Hall systems, J. Stat. Mech. L04001 (2006); Quantum Hall system in the Tao-Thouless limit, e-print arXiv:0712.1927v1 [cond-mat.mes-hall] (2007).

[BL] H.J. Brascamp and E.H. Lieb, Some inequalities for Gaussian measures and the long-range order of the one-dimensional plasma, Functional Integration and its Applications (A.M. Arthurs, ed.), 1975, pp. 1-14.

[BR] O. Bratteli and D.W. Robinson, Operator Algebras and Quantum Statistical Mechanics, vol. 1, Springer -Verlag, 1979.

[CFS] P. Choquard, P.J. Forrester, and E.R. Smith, The two-dimensional one-component plasma at $\Gamma=2$ : the semiperiodic strip, J. Stat. Phys. 33 (1983), 13-22.

[D] G.V. Dunne, Slater Decomposition of Laughlin States, Int. J. Mod. Phys. B 7 (1993), 4783-4813.

[Fel] W. Feller, An introduction to probability theory and its applications, 2 ed., vol. 1, John Wiley \& Sons, 1962.

[FGIL] P. Di Francesco, M. Gaudin, C. Itzykson, and F. Lesage, Laughlin's wave function, Coulomb gases and expansions of the discriminant, Int. J. Mod. Phys. A 9 (1994), 4287-4352.

[For] P.J. Forrester, Finite-Size Corrections to the Free Energy of Coulomb Systems with a Periodic Boundary Condition, J. Stat. Phys. 63 (1991), 491-504.

[GK] C. Gruber and H. Kunz, General properties of polymer systems, Commun. Math. Phys. 22 (1971), 133-161.

[HL] O.J. Heilmann and E.H. Lieb, Theory of Monomer-Dimer Systems, Commun. Math. Phys. 25 (1972), 190-232.

[HLP] G.H. Hardy, J.E. Littlewood, and G. Pólya, Inequalities, Cambridge University Press, 1964 .

[HR] F.D.M. Haldane and E.H. Rezayi, Periodic Laughlin-Jastrow wave functions for the fractional quantized Hall effect, Phys. Rev. B 31 (1985), 2529-2531.

[IVZ] D. Ioffe, Y. Velenik, and M. Zahradnik, Entropy-Driven Phase Transition in a Polydisperse Hard-Rods Lattice System, J. Stat. Phys. 122 (2006), 761-786.

[JL] B. Jancovici and J.L. Lebowitz, Bounded Fluctuations and Translation Symmetry Breaking: a Solvable Model, J. Stat. Phys. 103 (2001), 619-624.

[JLS] S. Jansen, E.H. Lieb and R. Seiler, Laughlin's function on a cylinder: plasma analogy and representation as a quantum polymer, phys. stat. sol. (b) 245 (2008), 439-446.

[KTW] R.C. King, F. Toumazet, and B.G. Wybourne, The square of the Vandermonde determinant and its q-generalisation, J. Phys. A 37 (2001), 737-767.

[K] H. Kunz, The One-Dimensional Classical Electron Gas, Ann. Phys. 85 (1974), 303-335.

[Lau1] R. B. Laughlin, Quantized Hall conductivity in two dimensions, Phys. Rev. B 23 (1981), 5632-5633.

[Lau2] R.B. Laughlin, Anomalous Quantum Hall Effect: An Incompressible Quantum Fluid with Fractionally Charged Excitations, Phys. Rev. Lett. 50 (1983), 13951398.

[Len] A. Lenard, Exact Statistical Mechanics of a One-Dimensional System with Coulomb Forces, J. Math. Phys. 2 (1961), 682-693.

[LL] J.L. Lebowitz and E.H. Lieb, Existence of Thermodynamics for Real Matter with Coulomb Forces, Phys. Rev. Lett. 22 (1969), 631 - 634. 
[LeeLein] D.-H. Lee and J.M. Leinaas, Mott Insulators without Symmetry Breaking, Phys. Rev. Lett. 92 (2004), 096401.

[RH] E.H. Rezayi and F.D.M. Haldane, Laughlin state on stretched and squeezed cylinders and edge excitations in the quantum Hall effect, Phys. Rev. B 50 (1994), 17199-17201.

[SFL] A. Seidel, H. Fu, D.-H. Lee, J.M. Leinaas, and J. Moore, Incompressible Quantum Liquids and New Conservation Laws, Phys. Rev. Lett 95 (2005), 266405.

[ŠWK] L. Šamaj, J. Wagner, and P. Kalinay, Translation Symmetry Breaking in the OneComponent Plasma on the Cylinder, J. Stat. Phys. 117 (2004), 159-178.

[T] D.J. Thouless, Theory of the quantized Hall effect, Surf. Sci. 142 (1984), 147-154.

[TSG] D.C. Tsui, H.L. Stormer, and A.C. Gossard, Two-Dimensional Magnetotransport in the Extreme Quantum Limit, Phys. Rev. Lett. 48 (1982), 1559 - 1562.

[TT] R. Tao and D.J. Thouless, Fractional quantization of Hall conductance, Phys. Rev. B 28 (1983), 1142-1144.

[TW] R. Tao and Y.-S. Wu, Gauge invariance and fractional quantum Hall effect, Phys. Rev. B 30 (1984), 1097-1098.

[WH] E. Westerberg and T.H. Hansson, Quantum mechanics on thin cylinders, Phys. Rev. B 47 (1993), 16554-16562. 